\title{
A MATHEMATICAL ANALYSIS OF ELECTRICAL DISCHARGES
}

\author{
BY \\ FRANCOIS SEVERIN (CRIL Technology, 2 impasse Henri Pitot, 31500 Toulouse, France and \\ CERMICS - INRIA, B. P. 9306902 Sophia-Antipolis, Cedex, France)
}

AND

ANNE NOURI (LATP, CMI, Université de Provence, 39 rue Joliot-Curie, 13453 Marseille Cedex 13, France)

\begin{abstract}
The study of systems arising in electrical discharges, via fluid models for charged and neutral particles is performed. Existence and uniqueness of the solution, locally in time, is proven for the drift-diffusion-Poisson system coupled with the isothermal Euler system, in a bounded domain, with the momentum prescribed on the boundary.
\end{abstract}

1. Introduction. The interest in the study of electrical discharges is getting more and more important nowadays (see [15], [16], and [5]). Discharges in a vacuum are analyzed in [2] and [14]. Electrical discharges also appear on satellites, due to the interaction with the solar wind. A better protection of satellites requires some knowledge of them. Two approaches are classically used. A kinetic approach, with the Vlasov equation for the electron distribution function [4] is used in [6], [10], and [11] in the study of discharges on solar arrays. A fluid approach, with a drift-diffusion equation for the electron density $([4],[7],[1])$ is used in this paper. Moreover, some numerical computations based on the model studied in this paper are performed in [20], providing simulations of electrical discharges in plasmas. The modelisation of a discharge is made with three kinds of particles: electrons, ions, and neutral molecules. The charged particles are governed by the drift-diffusion equations and the neutral ones by the isothermal Euler system. The coupling between the different types of particles stands in the ionization-recombination and the desorption phenomena.

Received February 28, 2000.

2000 Mathematics Subject Classification. Primary 35Q99, 65M25, 35K99, 35F25, 35F30.

E-mail address: francois.severin@criltechnology.com 
Denoting by $u$ the electron density, $v$ the ion density, $\rho$ the neutral density, $m$ its momentum, and $\Phi$ the electric potential, the following drift-diffusion system is studied:

$$
\left\{\begin{array}{l}
\partial_{t} u-\left(D_{e} u_{x}-\mu_{e} u \Phi_{x}\right)_{x}=\left(\nu_{i}\left(t, x, \rho, \Phi_{x}\right)-r v\right) u \text { in } P \\
\partial_{t} v-\left(D_{i} v_{x}+\mu_{i} v \Phi_{x}\right)_{x}=\left(\nu_{i}\left(t, x, \rho, \Phi_{x}\right)-r v\right) u \text { in } P \\
\Phi_{x x}=e(u-v) \text { in } P \\
D_{e} u_{x}=\mu_{e} u \Phi_{x} \text { on } \Sigma \\
v=0 \text { on } \Sigma \\
\Phi=g \text { on } \Sigma \\
u(0, \cdot)=u_{0} \\
v(0, \cdot)=v_{0}
\end{array}\right.
$$

coupled with the isothermal Euler system

$$
\left\{\begin{array}{l}
-\lambda_{2}(\rho, m)\left(\rho_{t}+\lambda_{1}(\rho, m) \rho_{x}\right)+m_{t}+\lambda_{1}(\rho, m) m_{x}=0 \\
-\lambda_{1}(\rho, m)\left(\rho_{t}+\lambda_{2}(\rho, m) \rho_{x}\right)+m_{t}+\lambda_{2}(\rho, m) m_{x}=0 \\
\rho(0, x)=\rho_{0}(x), \quad m(0, x)=m_{0}(x) \\
m(t, 0)=\mathcal{F}(t, 0):=\left(D_{i} v_{x}\right)(t, 0) \\
\rho(t, a)=\rho_{a}(t)
\end{array}\right.
$$

Here, $t$ and $x$ are respectively the time and the space variables, $P(\delta)=(0, \delta) \times(0, a)$ for a fixed time $\delta$ and a length $a$, and $\Sigma=(0, \delta) \times\{0, a\}$. The positive constants $D_{e}, D_{i}$ and the nonnegative constants $\mu_{e}$ and $\mu_{i}$ respectively denote the electron and ion diffusion and mobility. The functions $g, u_{0}, v_{0}, \rho_{a}, \rho_{0}$ and $m_{0}$ are given. Moreover,

$$
\lambda_{1}(\rho, m):=\frac{m}{\rho}-\alpha, \quad \lambda_{2}(\rho, m):=\frac{m}{\rho}+\alpha,
$$

$\alpha$ being a positive constant. On the one hand, one can recognize the ionization-recombination phenomena in the function $\left(\nu_{i}\left(t, x, \rho, \Phi_{x}\right)-r v\right) u$, where $\nu_{i}$ is the ionization frequency and $r$ is a positive constant. On the other hand, the desorption is described by Eq. (1.2.4). The ionization function $\nu_{i}$ is usually known by a range of experimental values. In this paper, a mathematical analysis provides conditions on the ionization function for having a well-posed problem. The mathematical complexity of the model lies in the coupling between the parabolic drift-diffusion equations and the hyperbolic Euler system satisfied by the charged and neutral particles respectively. Moreover, the boundary condition of vanishing flux does not allow any maximum principle for the electron density, contrary to [17], [18]. In Sec. 2, the existence and uniqueness of the solution to the drift-diffusion equations (1.1) is shown. Then in Sec. 3, the existence and uniqueness of the solution to the initial boundary Euler system is obtained in a setting introduced in [13]. It requires the boundary values $\mathcal{F}$ to be $\mathcal{C}^{1}$, i.e., the trace of $v_{x}$ to be $\mathcal{C}^{1}$. The regularity is controlled by using anisotropic Sobolev $H^{r, s}$ spaces. Finally, the existence and uniqueness of the solution to the whole system $(1.1,1.2)$ is obtained by a Schauder fixed point argument in Sec. 4.

In the whole paper, the ionization function $\nu:=\nu_{i}(t, x, \rho, E)$ is supposed to vanish at $(t, x, 0,0)$, as well as its two first derivatives in $t$ and in $x$, and to satisfy the following 
assumptions:

$$
\begin{aligned}
& \left|\frac{\partial^{k+l} \nu}{\partial t^{k} \partial x^{l}}(t, x, \sigma, E)-\frac{\partial^{k+l} \nu}{\partial t^{k} \partial x^{l}}(t, x, \tau, F)\right| \\
& \quad \leq c\left(\int_{0}^{t} \sup _{z}|(\sigma-\tau)(s, z)| d s+\left|\frac{\partial^{k+l}}{\partial t^{k} \partial x^{l}}(E-F)(t, x)\right|\right), \\
& (k, l) \in\{(0,0),(1,0),(2,0),(0,1),(0,2)\},
\end{aligned}
$$

and

$$
\begin{aligned}
& \left|\left(\frac{\partial^{l} \nu}{\partial x^{l}}(t, x, \sigma, E)-\frac{\partial^{l} \nu}{\partial x^{l}}(t, x, \tau, F)\right)-\left(\frac{\partial^{l} \nu}{\partial x^{l}}(t, y, \sigma, E)-\frac{\partial^{l} \nu}{\partial x^{l}}(t, y, \tau, F)\right)\right| \\
& \leq c\left(|x-y| \int_{0}^{t} \sup _{z}|(\sigma-\tau)(s, z)| d s+\left|\frac{\partial^{l}}{\partial x^{l}}(E-F)(t, x)-\frac{\partial^{l}}{\partial x^{l}}(E-F)(t, y)\right|\right) \\
& l \in\{0,1,2\}
\end{aligned}
$$

Here, $\nu(t, x, \sigma, E)\left(\right.$ resp. $\left.\nu_{x}(t, x, \sigma, E), \ldots\right)$ denotes $\nu(t, x, \sigma(t, x), E(t, x))$ (resp. $\nu_{x}(t, x$, $\sigma(t, x), E(t, x)), \ldots)$. An example of an ionization function $\nu$ satisfying these assumptions is given by

$$
\nu(t, x, \sigma, E)=E+\int_{0}^{t} \int_{0}^{s} \int_{0}^{x} \int_{0}^{x_{1}} \int_{0}^{x_{2}} \sigma(\alpha, \beta) d \alpha d \beta d x_{2} d x_{1} d s .
$$

2. Drift-diffusion equations coupled with Poisson equation. In this section, $\sigma$ is a fixed function in $C^{1}$. Denote by $\Omega=(0, a), \Gamma=\{0, a\}$ its boundary. Assume that $g \in L^{\infty}\left(0, T ; W^{\frac{7}{4}, 4}(\Gamma)\right)$ and denote by $C_{g}:=\|g\|_{L^{\infty}\left(0, T ; W^{\frac{7}{4}, 4}(\Gamma)\right)}$. Let $u_{0}$ and $v_{0}$ be nonnegative functions and $C_{K}$ a positive constant such that $\left\|u_{0}\right\|_{L^{p}(\Omega)}<C_{K}$ and $\left\|v_{0}\right\|_{L^{p}(\Omega)}<C_{K}$ for any $p \in[2,6]$. For any positive time $T$, let

$$
\widetilde{K}(T):=\left\{(u, v) \in L^{2}\left(0, T ; H^{1}(\Omega)\right) \times L^{2}\left(0, T ; H^{1}(\Omega)\right) ; 0 \leq u, 0 \leq v\right\}
$$

and

$$
K(T):=\left\{(u, v) \in \widetilde{K} ;\|u\|_{L^{\infty}\left(0, T ; L^{4}(\Omega)\right)} \leq C_{K},\|v\|_{L^{\infty}\left(0, T ; L^{4}(\Omega)\right)} \leq C_{K}\right\} .
$$

$K(T)$ is a closed and convex subset of $L^{2}\left(0, T, L^{4}(\Omega)\right) \times L^{2}\left(0, T, L^{4}(\Omega)\right)$.

TheOREM 1. There exists a positive time $T$ such that the system

$$
\left\{\begin{array}{l}
\partial_{t} u-\left(D_{e} u_{x}-\mu_{e} u \Phi_{x}\right)_{x}=\left(\nu_{i}\left(t, x, \sigma, \Phi_{x}\right)-r v\right) u, \quad(t, x) \in(0, T) \times \Omega \\
\partial_{t} v-\left(D_{i} v_{x}+\mu_{i} v \Phi_{x}\right)_{x}=\left(\nu_{i}\left(t, x, \sigma, \Phi_{x}\right)-r v\right) u, \quad(t, x) \in(0, T) \times \Omega \\
\Phi_{x x}=e(u-v), \quad(t, x) \in(0, T) \times \Omega \\
D_{e} u_{x}=\mu_{e} u \Phi_{x}, \quad(t, x) \in(0, T) \times \Gamma \\
v=0, \quad(t, x) \in(0, T) \times \Gamma \\
\Phi=g, \quad(t, x) \in(0, T) \times \Gamma \\
u(0, x)=u_{0}(x), \quad x \in \Omega \\
v(0, x)=v_{0}(x), \quad x \in \Omega
\end{array}\right.
$$

has a unique solution $((u, v), \Phi) \in K(T) \times L^{2}\left(0, T, W^{2,2}(\Omega)\right)$. 
Proof. A fixed point method is used. Let us first recall some results about the solution to the Poisson equation with a Dirichlet condition (see [8], [3]). Let $f \in L^{p}(\Omega)$ and $V_{d} \in W^{2-\frac{1}{p}, p}(\Gamma)$. Then the solution $V$ to

$$
\begin{gathered}
V_{x x}=f \quad \text { in } \Omega, \\
V=V_{d} \quad \text { on } \Gamma
\end{gathered}
$$

belongs to $V \in W^{2, p}(\Omega)$, and there exists a constant $C_{2, p}$ such that

$$
\|V\|_{W^{2, p}(\Omega)} \leq C_{2, p}\left(\|f\|_{L^{2}}+\left\|V_{d}\right\|_{W^{2-\frac{1}{p}, p}(\Gamma)}\right) .
$$

Moreover, by a Sobolev continuous imbedding, there exists a constant $C_{i n j}$ such that

$$
\left\|V_{x}\right\|_{L^{\infty}(\Omega)} \leq C_{\text {inj }}\|V\|_{W^{2,4}(\Omega)} .
$$

By assumption on $\nu$, there are constants $A_{1}$ and $A_{2}$ such that

$$
\nu(t, x, \sigma, E) \leq A_{1} E+A_{2} t .
$$

Let $T$ be a time such that

$$
e^{\frac{T}{2}\left[A_{2} T^{2}+A_{1} C_{2,2} C_{i n j}\left(C_{K}+C_{g}\right)+\frac{\mu_{e}^{2}}{D_{e}} C_{K}^{2}+C_{g}^{2}\right]} \leq \frac{C_{k}}{\left\|u_{0}\right\|_{L^{4}(\Omega)}} .
$$

Let $F$ be the map

$$
\begin{aligned}
F: K(T) & \rightarrow K(T), \\
(u, v) & \mapsto(U, V),
\end{aligned}
$$

where $U$ and $V$ are the solutions to

$$
\begin{aligned}
\partial_{t} U-\left(D_{e} U_{x}-\mu_{e} U \Phi_{x}\right)_{x} & =\left(\nu_{i}\left(t, x, \sigma, \Phi_{x}\right)-r v\right) U, \quad(t, x) \in(0, T) \times \Omega, \\
\partial_{t} V-\left(D_{i} V_{x}+\mu_{i} V \Phi_{x}\right)_{x} & =\left(\nu_{i}\left(t, x, \sigma, \Phi_{x}\right)-r V\right) u, \quad(t, x) \in(0, T) \times \Omega, \\
D_{e} U_{x} & =\mu_{e} U \Phi_{x}, \quad(t, x) \in(0, T) \times \Gamma, \\
V & =0, \quad(t, x) \in(0, T) \times \Gamma,
\end{aligned}
$$

and $\Phi$ is the solution to

$$
\begin{aligned}
\Phi_{x x} & =e(u-v), \quad(t, x) \in(0, T) \times \Omega, \\
\Phi & =g, \quad(t, x) \in(0, T) \times \Gamma .
\end{aligned}
$$

Then

$$
\begin{aligned}
\left|\Phi_{x}(t, \cdot)\right|_{L^{\infty}(\Omega)} & \leq e C_{i n j}\|\Phi(t, \cdot)\|_{W^{2,4}(\Omega)} \\
& \leq e C_{i n j} C_{2,4}\left(\|u(t, \cdot)-v(t, \cdot)\|_{L^{4}(\Omega)}+C_{g}\right), \quad t<T .
\end{aligned}
$$

Hence $\left\|\Phi_{x}\right\|_{L^{\infty}(P(T))}$ is bounded from above uniformly with respect to $(u, v) \in K(T)$. There exists a unique solution $(U, V)$ to (2.6) that belongs to $\widetilde{K}(T)$ (see [19]). Using Gronwall's lemma and the definition of the time $T,\|U\|_{L^{\infty}\left(0, T ; L^{4}(\Omega)\right)} \leq C_{K}$. The same result holds for $V$. Thus $(U, V) \in K(T)$.

From now on, all constants will be denoted by $C$. Moreover, the map $F$ is compact. Indeed, $U$ and $V$ belong to

$$
\left\{f \in L^{2}\left(0, T, H^{1}\right), \partial_{t} f \in L^{2}\left(0, T, L^{2}\right)\right\},
$$


which imbeds compactly into $L^{2}\left(L^{4}\right)$. So, if the sequence $\left(u_{n}, v_{n}\right)$ is bounded in $K(T)$, a subsequence of $\left(U_{n}, V_{n}\right)=F\left(u_{n}, v_{n}\right) \in K(T)$ converges for the $L^{2}\left(L^{4}\right) \times L^{2}\left(L^{4}\right)$ topology to some $(U, V)$ belonging to $K(T)$. Then, the map $F$ is continuous for the $L^{2}\left(L^{4}\right) \times L^{2}\left(L^{4}\right)$ topology. Indeed, let

$$
\left(u_{n}, v_{n}\right) \stackrel{L^{2}\left(L^{4}\right)}{\longrightarrow}(u, v),
$$

and $\left(U_{n}, V_{n}\right)=F\left(u_{n}, v_{n}\right),(U, V)=F(u, v)$. Denote by $\Phi_{n}:=\Phi\left(u_{n}, v_{n}\right), \nu_{i}:=\nu_{i}(t, x, \sigma$, $\left.\Phi_{x}\right), \nu_{i}^{n}:=\nu_{i}\left(t, x, \sigma, \Phi_{n x}\right), w:=U_{n}-U, \widetilde{\Phi}=\Phi_{n}-\Phi$. They satisfy

$$
\left\{\begin{array}{l}
w_{t}-\left(D_{e} w_{x}-\mu_{e} w \Phi_{n x}+\mu_{e} U\left(\Phi-\Phi_{n}\right)_{x}\right)_{x} \\
\quad=\left(\nu_{i}^{n}-r v_{n}\right) U_{n}-\left(\nu_{i}-r v\right) U, \quad(t, x) \in(0, T) \times \Omega, \\
D_{e} w_{x}=\mu_{e} U_{n} \Phi_{n x}-\mu_{e} U \Phi_{x}, \quad(t, x) \in(0, T) \times \Gamma, \\
w(0, x)=0, \quad x \in \Omega,
\end{array}\right.
$$

and the Poisson equation

$$
\begin{aligned}
\bar{\Phi}_{x x} & =e\left(u_{n}-u-\left(v_{n}-v\right)\right), \quad(t, x) \in(0, T) \times \Omega, \\
\bar{\Phi} & =0, \quad(t, x) \in(0, T) \times \Gamma .
\end{aligned}
$$

Denote by $\bar{u}:=u_{n}-u, \bar{v}:=v_{n}-v$. It is easy to see that

$$
\begin{aligned}
\frac{1}{2} \int_{\Omega} w^{2}(t, x) d x+\frac{D_{e}}{2} \int_{P(t)}\left|w_{x}\right|^{2} \leq & C \int_{0}^{t}\left[f(s) \int_{\Omega} w^{2}(s, x) d x\right. \\
& +\|(\bar{u}-\bar{v})(s, \cdot)\|_{L^{4}}^{2}\left(C+\|U(s, \cdot)\|_{L^{2}}^{2}\right) \\
& \left.+\|\bar{v}(s, \cdot)\|_{L^{2}}^{2}\left\|\bar{U}_{n}(s, \cdot)\right\|_{L^{\frac{7}{12}}}^{2}\right] d s,
\end{aligned}
$$

with $f(s)=C\left|\Phi_{n x}(s, \cdot)\right|_{\infty}^{2}+\left\|U_{n}(s, \cdot)\right\|_{L^{2}}^{2}+C+\left|\nu_{i}\right|$. A similar inequality holds for the ions. The difference $z=V_{n}-V$ satisfies

$$
\begin{aligned}
& \frac{1}{2} \int_{\Omega} z^{2}(t, x) d x+\frac{D_{i}}{2} \int_{P(t)}\left|z_{x}\right|^{2} \\
& \quad \leq C \int_{0}^{t}\left[f(s) \int_{\Omega} z^{2}(s, x) d x+\|(\bar{u}-\bar{v})(s, \cdot)\|_{L^{4}}^{2} \int_{\Omega} V^{2}(s, x) d x\right] d s .
\end{aligned}
$$

Hence,

$$
\begin{aligned}
\frac{1}{2} \int_{\Omega}\left(w^{2}(t, x)+z^{2}(t, x)\right) d x+\int_{P(t)}\left(\left|w_{x}\right|^{2}+\left|z_{x}\right|^{2}\right) \leq & C \int_{0}^{t}\left[f(s) \int_{\Omega}\left(w^{2}(s, x)+z^{2}(s, x)\right) d x\right. \\
& \left.+\|(\bar{u}-\bar{v})(s, \cdot)\|_{L^{4}}^{2}\right] d s,
\end{aligned}
$$

so that, by Gronwall's lemma,

$$
\int_{\Omega}\left(w^{2}+z^{2}\right)(t, x) d x \leq C\left(\int_{0}^{t}\|\bar{u}-\bar{v}\|_{L^{4}}^{2}\right) e^{\int_{0}^{t} f(s) d s} .
$$

There is a similar result for $\int_{P(t)}\left(\left|w_{x}\right|^{2}+\left|z_{x}\right|^{2}\right)$. The boundedness of $\int_{0}^{t} f(s) d s$ leads to the continuity of the map $F$. The Schauder fixed point theorem implies the existence of a solution $(u, v, \Phi)$ of the system (2.3). For establishing the uniqueness of the solution to 
(2.3), let $\left(u_{1}, v_{1}, \Phi_{1}\right)$ and $\left(u_{2}, v_{2}, \Phi_{2}\right)$ be two solutions of the system (2.3). The differences $w=u_{1}-u_{2}, z=v_{1}-v_{2}$, and $\bar{\Phi}=\Phi_{1}-\Phi_{2}$ satisfy

$$
\begin{aligned}
& \partial_{t} w-\left(D_{e} w_{x}-\mu_{e} w \Phi_{1 x}+\mu_{e} u_{2} \bar{\Phi}_{x}\right)_{x}=\bar{\nu}_{i} u_{1}+\nu_{i}^{2} w-r w v_{1}-r u_{2} z \quad \text { in } P(T), \\
& D_{e} w_{x}-\mu_{e} w \Phi_{1 x}+\mu_{e} u_{2} \bar{\Phi}_{x}=0 \quad \text { on } \Sigma, \\
& w(0, x)=0, \quad x \in \Omega,
\end{aligned}
$$

where $\bar{\nu}:=\nu_{i}\left(t, x, \sigma, \Phi_{1}\right)-\nu_{i}\left(t, x, \sigma, \Phi_{2}\right)$.

With similar computations to previously, and using Gronwall's lemma, the uniqueness of the solution $(u, v, \Phi)$ to $(2.3)$ follows.

REMARKS.

- There exists a maximum principle for $v$. It can easily be seen that if $N$ is a constant such that $v_{0} \leq N$ and $N\left(r-\mu_{i}\right)-\left\|\nu_{i}\right\|_{\infty} \geq 0$, then $v \leq N$.

- There is no maximum principle for $u$. On account of the boundary condition, it is difficult to expand

$$
\left(D_{e} u_{x}-\mu_{e} u \Phi_{x}\right)_{x},
$$

which is classically done for getting a maximum principle in the case of a Dirichlet condition on the boundary.

\section{The Euler system.}

3.1. The Cauchy problem.

Theorem 2. Assume that the initial conditions $\rho_{0}$ and $m_{0}$ belong to $\mathcal{C}^{1}$ and satisfy $0<\beta \leq \rho_{0}(0) \leq \Omega^{c},\left|m_{0}(0)\right| \leq \Omega^{c}$ for some constants $\beta$ and $\Omega^{c}$. Then, there exists a positive time $\delta$ such that the system $(1.2 .1,1.2 .2,1.2 .3)$ has a unique solution $\left(\rho^{c}, m^{c}\right)$ in $\mathcal{C}^{1}(Q(\delta))$ where $Q(\delta)$ is defined by

$$
Q(\delta):=\left\{0 \leq t \leq \delta, x_{1}(t) \leq x \leq x_{2}(t)\right\},
$$

the curves $x_{1}$ and $x_{2}$ being defined, as characteristics of the Euler system, by

$$
\left\{\begin{array}{l}
\frac{d x_{1}(s)}{d s}=\lambda_{2}\left(s, x_{1}, \rho^{c}\left(s, x_{1}\right), m^{c}\left(s, x_{1}\right)\right), \\
x_{1}(0)=0
\end{array}\right.
$$

and

$$
\left\{\begin{array}{l}
\frac{d x_{2}(s)}{d s}=\lambda_{1}\left(s, x_{2}, \rho^{c}\left(s, x_{2}\right), m^{c}\left(s, x_{2}\right)\right), \\
x_{2}(0)=a .
\end{array}\right.
$$

Proof. It is based on a fixed point method of Schauder type. Following Li Ta-tsien and $\mathrm{Yu}$ Wen-ci [13], a constant $\Omega_{1}^{c}$ and a function $\Omega_{2}(\eta)$ tending to zero when $\eta$ tends to zero are determined. Then, define the set $\Sigma_{0}^{c}\left(\delta \mid \Omega^{c}, \Omega_{1}^{c}, \Omega_{2}^{c}(\cdot)\right)$ by

$$
\Sigma_{0}^{c}\left(\delta \mid \Omega^{c}, \Omega_{1}^{c}, \Omega_{2}^{c}(\cdot)\right):=\left\{v \in \Sigma_{0}^{c}\left(\delta \mid \Omega^{c}, \Omega_{1}^{c}\right), \omega(\eta, q)+\omega(\eta, z) \leq \Omega_{2}^{c}(\eta), \eta>0\right\},
$$


where $q:=v_{t}, z=v_{x}, \omega$ is the modulus of continuity, and the set $\Sigma_{0}^{c}\left(\delta \mid \Omega^{c}, \Omega_{1}^{c}\right)$ is defined by

$$
\Sigma_{0}^{c}\left(\delta \mid \Omega^{c}, \Omega_{1}^{c}\right)=\left\{v \in\left(C^{1}(Q(\delta))\right)^{2}, \frac{\beta}{2} \leq v_{1} \leq \Omega^{c},\left|v_{2}\right| \leq \Omega^{c},\|v\|_{1} \leq \Omega_{1}^{c}\right\} .
$$

The set $\Sigma_{0}^{c}\left(\delta \mid \Omega^{c}, \Omega_{1}^{c}, \Omega_{2}^{c}(\cdot)\right)$ is a compact subset of $\mathcal{C}^{0}(Q(\delta))$. Then a Schauder fixed point argument is used in $\Sigma_{0}^{c}\left(\delta \mid \Omega^{c}, \Omega_{1}^{c}, \Omega_{2}^{c}(\cdot)\right)$.

3.2. The boundary value problems. Define the subspaces $R(\delta)$ and $S(\delta)$ by

$$
\begin{aligned}
R(\delta) & :=\left\{0 \leq t \leq \delta, 0 \leq x \leq x_{1}(t)\right\}, \\
S(\delta) & :=\left\{0 \leq t \leq \delta, x_{2}(t) \leq x \leq a\right\} .
\end{aligned}
$$

TheOREM 3. Assume that the boundary condition $\mathcal{F}$ belongs to $\mathcal{C}^{1}(R(\delta))$. Assume $\mathcal{F}>0$ and $\lambda_{1}^{0}<0$. Then there exists a unique solution $(\rho, m)$ to $(1.2 .1,1.2 .2,1.2 .4)$ that belongs to $\mathcal{C}^{1}(R(\delta))$.

Proof. By the assumption, the minimal characterizing number $\theta_{\min }$ defined in Li Tatsien and Yu Wen-ci [13] by

$$
\theta_{\min }:=\inf _{\gamma_{i} \neq 0} \max _{l=1,2} \sum_{k=1}^{2}\left|\sum_{j=1}^{2} \frac{\gamma_{l}}{\gamma_{k}} H_{k l}\right|
$$

where

$$
H_{11}=H_{12}=0, \quad H_{22}=-H_{21}=\frac{\lambda_{1}^{0}}{\lambda_{1}^{0}-\lambda_{2}^{0}}
$$

satisfies $\theta_{\min }<1$. Then, similarly to $\Sigma_{0}^{c}\left(\delta \mid \Omega^{c}, \Omega_{1}^{c}, \Omega_{2}^{c}(\cdot)\right)$, a convex compact subset $\Sigma^{p b l}(R(\delta))$ of $\mathcal{C}^{0}(R(\delta))$ is defined by

$$
\Sigma^{p b l}(R(\delta)):=\left\{v \in\left(C^{1}(\bar{R}(\delta))\right)^{2} ; v(0,0)=0,\|q\| \leq \Omega_{1}, \Omega(\eta, q) \leq \Omega_{2}(\eta)\right\}
$$

where

$$
q_{i}=\partial_{t} v_{i}, \quad q_{2+i}=\partial_{t} v_{i}+\partial_{x} v_{i}, \quad i=1,2
$$

and

$$
\Omega(\eta, f)=\sup _{\left|t-t^{\prime}\right| \leq \eta,\left|x-x^{\prime}\right| \leq \eta,(t, x) \in \bar{R}(\delta),\left(t^{\prime}, x^{\prime}\right) \in \bar{R}(\delta)}\left|f(t, x)-f\left(t^{\prime}, x^{\prime}\right)\right| .
$$

Hence, following [13], a fixed point theorem can be applied in $\Sigma^{p b l}(R(\delta))$, so that there exists a unique solution to (4.27) that belongs to $\mathcal{C}^{1}(R(\delta))$ for $\delta$ small enough.

In a similar way, one can prove the following.

TheOrem 4. Assume that the boundary condition $\rho_{a}$ belongs to $\mathcal{C}^{1}(R(\delta))$ and that $\lambda_{2}^{0}>0$. Then there exists a unique solution to $(1.2 .1,1.2 .2,1.2 .5)$ that belongs to $\mathcal{C}^{1}(S(\delta))$. 
4. The whole problem. Let $\delta_{0}$ be a positive constant. For the definition and the main properties of the anisotropic spaces $H^{r, s}(P(\delta))\left(\delta \leq \delta_{0}\right)$ we refer to [12] and [9].

TheOREm 5. Assume $u_{0}, v_{0} \in W^{2,2}(\Omega), 0 \leq u_{0}, 0 \leq v_{0}$, and $g \in L^{\infty}\left(0, \delta_{0} ; H^{\frac{11}{2}}(\Gamma)\right) \cap$ $H^{3}\left(0, \delta_{0} ; H^{\frac{5}{2}}(\Gamma)\right) \cap W^{3, \infty}\left(0, \delta_{0} ; H^{\frac{3}{2}}(\Gamma)\right)$. Assume that there are two constants $\beta$ and $\Omega^{c}$ such that $\rho_{0}$ and $m_{0}$ satisfy $0<\beta \leq \rho_{0}(0) \leq \Omega^{c},\left|m_{0}(0)\right| \leq \Omega^{c}$, and

$$
\frac{m_{0}(0)}{\rho_{0}(0)}-\alpha<0<\frac{m_{0}(0)}{\rho_{0}(0)}+\alpha \text {. }
$$

Then there is a positive time $\delta$ such that the systems (1.1-1.2) have a unique solution $(u, v, \Phi, \rho, m)$ that belongs to $H^{\frac{19}{4}, \frac{19}{8}}(P(\delta)) \times H^{\frac{19}{4}, \frac{19}{8}}(P(\delta)) \times L^{2}\left(0, \delta ; H^{\frac{27}{4}}(\Omega)\right) \times$ $\mathcal{C}^{1}((P(\delta))) \times \mathcal{C}^{1}((P(\delta)))$.

Proof. A fixed point method of Schauder type will be used to prove the existence and uniqueness of the solution to the system (1.1-1.2) in the compact convex subset $\Sigma(P(\delta))$ defined by

$$
\Sigma(P(\delta)):=\left\{v ; v_{\mid Q(\delta)} \in \Sigma_{0}^{c}\left(\delta \mid \Omega^{c}, \Omega_{1}^{c}, \Omega_{2}^{c}(\cdot)\right), v_{\mid R(\delta)} \in \Sigma^{p b l}(R(\delta)), v_{\mid S(\delta)} \in \Sigma^{p b l}(S(\delta))\right\} .
$$

Let $\mathcal{T}$ be the map

$$
\begin{aligned}
\mathcal{T}: \Sigma(P(\delta)) & \rightarrow \Sigma(P(\delta)), \\
\sigma & \mapsto \rho:=\mathcal{T}(\sigma),
\end{aligned}
$$

where $\rho$ is the first component of the solution $(\rho, m)$ to

$$
\left\{\begin{array}{l}
-\lambda_{2}\left(\rho_{t}+\lambda_{1} \rho_{x}\right)+m_{t}+\lambda_{1} m_{x}=0 \\
-\lambda_{1}\left(\rho_{t}+\lambda_{2} \rho_{x}\right)+m_{t}+\lambda_{2} m_{x}=0 \\
\rho(0, x)=\rho_{0}(x), \quad m(0, x)=m_{0}(x), \\
m(t, 0)=\mathcal{F}(t, 0)=\left(D_{i} v_{x}\right)(t, 0), \\
\rho(t, a)=\rho_{a}(t),
\end{array}\right.
$$

and where $v$ and $\Phi$ are the solutions to

$$
\left\{\begin{array}{l}
\partial_{t} u-\left(D_{e} u_{x}-\mu_{e} u \Phi_{x}\right)_{x}=\left(\nu_{i}\left(t, x, \sigma, \Phi_{x}\right)-r v\right) u \text { in } Q \\
\partial_{t} v-\left(D_{i} v_{x}+\mu_{i} v \Phi_{x}\right)_{x}=\left(\nu_{i}\left(t, x, \sigma, \Phi_{x}\right)-r v\right) u \text { in } Q \\
\Phi_{x x}=e(u-v) \text { in } Q \\
D_{e} u_{x}=\mu_{e} u \Phi_{x} \text { in } \Sigma \\
v=0 \text { in } \Sigma \\
\Phi=g \text { in } \Sigma \\
u(0, \cdot)=u_{0} \\
v(0, \cdot)=v_{0}
\end{array}\right.
$$

We will see in Subsection 4.3 that $\mathcal{T}$ maps $\Sigma(P(\delta))$ into itself. 
Since $\Sigma(P(\delta))$ is a compact and convex subset of $C^{0}$, the continuity of $\mathcal{T}$ is a sufficient condition for having a fixed point. The map $\mathcal{T}$ splits into two maps,

$$
\begin{gathered}
\Sigma(P(\delta)) \stackrel{\mathcal{T}_{1}}{\longrightarrow} \mathcal{C}^{1}([0, \delta]) \stackrel{\mathcal{T}_{2}}{\longrightarrow} \Sigma(P(\delta)), \\
\sigma \longmapsto \\
\mathcal{F} \longmapsto
\end{gathered}
$$

4.1. Continuity of the map $\mathcal{T}_{1}$. For an easier reading, $D_{e}, \mu_{e}, D_{i}, \mu_{i}$ and $e$ are taken equal to 1 . Thanks to Theorem 1 , there exists a time $T\left(T \leq \delta_{0}\right)$ such that there exists a unique solution $(u, v, \Phi)$ that belongs to $L^{2}\left(0, T ; H^{1}(\Omega)\right)^{2} \times L^{\infty}\left(0, T ; W^{2,2}(\Omega)\right)$. In the following, the regularity of $u$ and $v$ is detailed, and then the continuity of $\mathcal{T}_{1}$ is proven.

Let $(u, v, \Phi)$ be the solution to the Drift-Diffusion-Poisson equations written in the form

$$
\left\{\begin{array}{l}
\partial_{t} u-u_{x x}=f_{e}:=\nu_{i}\left(t, x, \sigma, \Phi_{x}\right) u-r u v-\left(u \Phi_{x}\right)_{x} \quad \text { in } P(T), \\
\partial_{t} v-v_{x x}=f_{i}:=\nu_{i}\left(t, x, \sigma, \Phi_{x}\right) u-r u v+\left(v \Phi_{x}\right)_{x} \text { in } P(T), \\
u_{x}=u \Phi_{x} \text { on } \Sigma \\
v=0 \text { on } \Sigma \\
\Phi_{x x}=u-v \text { in } P(T), \\
\Phi=g \text { on } \Sigma .
\end{array}\right.
$$

Denote by $E:=\Phi_{x}$. By the first equation of (2.3), the initial condition for $u_{t}$ is

$$
u_{t}(0, x)=\nu_{i}\left(0, x, \rho_{0}(x), E(0, x)\right) u_{0}(x)-r u_{0}(x) v_{0}(x)+\partial_{x}\left(\partial_{x} u_{0}(x)-u_{0}(x) \partial_{x} \Phi(0, x)\right) .
$$

Since $u_{0}$ and $\Phi(0, x)$ belong to $H^{2}(\Omega), u_{t}(0, x) \in L^{2}(\Omega)$.

LEMma 6. If $g_{t} \in L^{2}\left(0, T ; H^{\frac{3}{2}}(\Gamma)\right)$, then $u_{t}, v_{t} \in L^{2}\left(0, T ; H^{1}(\Omega)\right) \cap L^{\infty}\left(0, T ; L^{2}(\Omega)\right)$.

Lemma 7. If $g_{t t} \in L^{2}\left(0, T ; H^{\frac{3}{2}}(\Gamma)\right)$, then $u_{t t}, v_{t t} \in L^{2}\left(0, T ; H^{1}(\Omega)\right) \cap L^{\infty}\left(0, T ; L^{2}(\Omega)\right)$.

Notation. For any function $G$ defined on $\Gamma$, denote by $\left.G\right|_{\Gamma}:=(G(0), G(a))$. For any function $G$ defined on $\Sigma$, denote by $\left.G\right|_{\Sigma}:=(G(t, 0), G(t, a)), t \in(0, T)$. Denote also by $g_{e}:=u \Phi_{x}$.

ThEOREM 8. Let $u_{0} \in H^{4}(\Omega), v_{0} \in H^{4}(\Omega)$ be such that

$$
\begin{array}{ll}
u_{0}^{(p)}(0)=u_{0}^{(p)}(a)=0, & p \in\{0,1,2,3\}, \\
v_{0}^{(p)}(0)=v_{0}^{(p)}(a)=0, & p \in\{0, \ldots, 4\} .
\end{array}
$$

Assume that the Dirichlet data $g$ for the potential $\Phi$ satisfy

$$
g \in \mathcal{C}^{0}\left(0, T ; H^{\frac{11}{2}}(\Gamma)\right) \cap H^{3}\left(0, T ; H^{\frac{5}{2}}(\Gamma)\right) \cap W^{3, \infty}\left(0, T ; H^{\frac{3}{2}}(\Gamma)\right) .
$$

Then $\partial_{x} u(\cdot, 0), \partial_{x} u(\cdot, a), \partial_{x} v(\cdot, 0)$, and $\partial_{x} v(\cdot, a)$ belong to $\mathcal{C}^{1}([0, T])$, where $(u, v)$ is the solution to (4.17). 
Proof of Theorem 8. The proof consists of five steps.

(i) In this step, the belongness of $u$ and $v$ to $H^{\frac{7}{4}, \frac{7}{8}}(P(T))$ is proven. It is sufficient to prove $f_{e}, f_{i} \in L^{2}, g_{e} \in H^{\frac{1}{4}}(\Gamma)$, and the compatibility relations [12]

$$
\frac{\partial u_{0}}{\partial \nu}(x)=u_{0}(x) \frac{\partial \Phi(0, x)}{\partial \nu}(x), \quad x \in \Gamma .
$$

First, $f_{e}$ and $f_{i}$ belong to $L^{2}$ since $u$ and $v$ belong to $L^{2}\left(H^{1}\right)$. Then, $\left.u \Phi_{x}\right|_{\Sigma} \in H^{\frac{1}{4}}(] 0, T[$ $\times \Gamma)$. Indeed,

$$
\left\|u \Phi_{x}\right\|_{H^{1}\left(L^{2}\right)}^{2}=\left\|u \Phi_{x}\right\|_{L^{2}\left(L^{2}\right)}^{2}+\left\|u_{t} \Phi_{x}+u \Phi_{t x}\right\|_{L^{2}\left(L^{2}\right)}^{2} .
$$

The integral $\int_{P(T)} u_{t}^{2} \Phi_{x}^{2}$ is bounded because $\Phi_{x}$ is bounded and $u_{t} \in L^{2}\left(H^{1}\right)$. Then, it follows from Lemma 6 and the properties of Poisson's equation, that $\Phi$ and $\Phi_{t}$ belong to $L^{2}\left(0, T ; H^{3}(\Omega)\right) \cap L^{\infty}\left(0, T ; H^{2}(\Omega)\right)$. Thus

$$
\int_{P(T)} u^{2} \Phi_{t x}^{2} \leq \int_{0}^{T}\|u\|_{H^{1}}^{2}\left\|\Phi_{t}\right\|_{H^{2}}^{2} .
$$

Thus $u \Phi_{x} \in H^{1,1}$ and $u \Phi_{x} \in H^{1, \frac{1}{2}}$, and so, $\left(u \Phi_{x}\right)_{\Sigma} \in H^{\frac{1}{4}}((0, T) \times \Gamma)$.

The compatibility condition for $u$ holds by (4.18). The compatibility condition for $v$, which writes $v_{0}(x)=0, x \in \Gamma$, follows from (4.19). Hence, by Theorem 6.2 of [12],

$$
u, v \in H^{\frac{7}{4}, \frac{7}{8}}(P(T)) .
$$

(ii) In this step, the belongness of $u$ and $v$ to $H^{\frac{11}{4}, \frac{11}{8}}(P(T))$ is proven. It is sufficient to prove $f_{e}, f_{i} \in H^{\frac{3}{4}}, \frac{3}{8}, g_{e} \in H^{\frac{5}{8}}(\Sigma)$, and the compatibility relations

$$
\left(u(0, \cdot) \Phi_{x}(0, \cdot)\right)_{\Gamma}=u_{x}(0, \cdot)_{\Gamma} .
$$

Similar computations to Step 1 show that $f_{e}, f_{i} \in H^{\frac{3}{4}, \frac{3}{8}}$. The belongness of $g_{e}$ to $H^{\frac{5}{8}}(\Sigma)$ is proven as follows. For $s=\frac{5}{8}$,

$$
\begin{aligned}
\left\|g_{e}\right\|_{H^{s}(0, T)}^{2}= & \int_{0}^{T} \int_{0}^{T} \frac{\left|\left(u \Phi_{x}\right)(t, 0)-\left(u \Phi_{x}\right)\left(t^{\prime}, 0\right)\right|^{2}}{\left|t-t^{\prime}\right|^{2 s+1}} d t d t^{\prime} \\
& +\int_{0}^{T} \int_{0}^{T} \frac{\left|\left(u \Phi_{x}\right)(t, a)-\left(u \Phi_{x}\right)\left(t^{\prime}, a\right)\right|^{2}}{\left|t-t^{\prime}\right|^{2 s+1}} d t d t^{\prime} \\
\leq & \int_{0}^{T} \int_{0}^{T} \frac{\left|\left(u(t, 0)-u\left(t^{\prime}, 0\right)\right) \Phi_{x}(t, 0)\right|^{2}+\left|u\left(t^{\prime}, 0\right)\left(\Phi_{x}(t, 0)-\Phi_{x}\left(t^{\prime}, 0\right)\right)\right|^{2}}{\left|t-t^{\prime}\right|^{2 s+1}} d t d t^{\prime} \\
& +\int_{0}^{T} \int_{0}^{T} \frac{\left|\left(u(t, a)-u\left(t^{\prime}, a\right)\right) \Phi_{x}(t, a)\right|^{2}+\left|u\left(t^{\prime}, a\right)\left(\Phi_{x}(t, a)-\Phi_{x}\left(t^{\prime}, a\right)\right)\right|^{2}}{\left|t-t^{\prime}\right|^{2 s+1}} d t d t^{\prime} .
\end{aligned}
$$

It follows from the boundedness of $\Phi$ in $H^{\frac{15}{4}, 1}(0, T ; \Omega)$ that $\left|\Phi_{x}(t, 0)\right|$ and $\left|\Phi_{x}(t, a)\right|$ are bounded by $\|\Phi\|_{H^{\frac{15}{4}, 1}(0, T ; \Omega)}$. Moreover, $u \in \mathcal{C}^{0}\left([0, T] ; H^{\frac{3}{4}}\right) \subset \mathcal{C}^{0}([0, T] ; \bar{\Omega})$, and so,

$$
\begin{aligned}
\left\|g_{e}\right\|_{H^{\frac{5}{8}}(0, T)}^{2} & \leq C\|u\|_{H^{\frac{5}{8}}(0, T)}^{2}+C\left\|\left(\Phi_{x}\right)_{\Gamma}\right\|_{H^{\frac{5}{8}}(0, T)}^{2} \\
& \leq C\|u\|_{H^{\frac{7}{4} \cdot \frac{7}{8}(0 . T: \Omega)}}^{2}+C\left\|\Phi_{x}\right\|_{H^{\frac{7}{4}} \cdot \frac{7}{8}(0, T ; \Omega)}^{2} .
\end{aligned}
$$

It is easy to see that $\Phi_{x} \in L^{2}\left(0, T ; H^{\frac{7}{4}}(\Omega)\right)$. Then $\Phi_{x} \in H^{\frac{7}{8}}\left(0, T ; L^{2}(\Omega)\right)$. Thus $g_{e} \in$ $H^{\frac{5}{8}}(0, T)$. By $(4.18)$, the compatibility relations are satisfied. It follows from Theorem 6.2 of [12] that $u, v \in H^{\frac{11}{4}, \frac{11}{8}}(P(T))$. 
(iii) The belongness of $u$ and $v$ to $H^{\frac{15}{4}, \frac{15}{8}}(P(T))$ is proven as in Steps 1 and 2 .

(iv) The belongness of $u$ and $v$ to $H^{\frac{19}{4}, \frac{19}{8}}(P(T))$ is proven as in the previous steps.

(v) It follows from step (iv) that

$$
u(\cdot, 0) \in H^{\beta_{0}}(0, T), u(\cdot, a) \in H^{\beta_{0}}(0, T), \partial_{x} u(\cdot, 0) \in H^{\beta_{1}}(0, T), \partial_{x} u(\cdot, a) \in H^{\beta_{1}}(0, T),
$$

with $\beta_{0}=\frac{17}{8}$ and $\beta_{1}=\frac{13}{8}$, respectively. Hence

$$
\begin{aligned}
u(\cdot, 0) & \in \mathcal{C}^{1}([0, T]), & & u(\cdot, a) \in \mathcal{C}^{1}([0, T]), \\
u_{x}(\cdot, 0) & \in \mathcal{C}^{1}([0, T]), & & u_{x}(\cdot, a) \in \mathcal{C}^{1}([0, T]),
\end{aligned}
$$

and similar regularities hold for $v(\cdot, 0), v_{x}(\cdot, 0), v(\cdot, a)$, and $v_{x}(\cdot, a)$. This ends the proof of Theorem 8.

Corollary 9. Under the hypothesis of Theorem 8, the flux and the ions at the boundary,

$$
\mathcal{F}(t):=\partial_{x} v(t, 0)+v(t, 0) \partial_{x} \Phi(t, 0)
$$

belongs to $\mathcal{C}^{1}([0, T])$.

Proof. The boundary condition for the ions, namely $v=0$ on $\Sigma$, leads to $\mathcal{F}(t)=$ $\partial_{x} v(t, 0)$. The result then follows from Theorem 8 .

Theorem 10. For any $\delta \leq T$, the map

$$
\mathcal{T}_{1}: \sigma \mapsto \mathcal{F}
$$

is continuous from $\Sigma(P(\delta))$ into $C^{1}([0, \delta])$.

Proof. Let $\left(\sigma^{k}\right)$ be a sequence in $\Sigma(P(\delta))$ tending to some $\sigma$ in $\mathcal{C}^{0}$, and let $\left(E^{k}\right)$ and $E$ be the electric fields respectively associated to $\left(\sigma^{k}\right)$ and $\sigma$ in (4.16). Denote by $\bar{\sigma}$ and $\bar{E}$, respectively,

$$
\bar{\sigma}(t, x):=\sigma(t, x)-\sigma^{k}(t, x), \quad \bar{E}(t, x):=E(t, x)-E^{k}(t, x) .
$$

Let $u, u_{k}, v, v_{k}$ be the solutions of the drift-diffusion-Poisson system (4.17). Denote by - all expressions with differences. For the electrons,

$$
\begin{aligned}
\partial_{t} \bar{u}-\bar{u}_{x x}=\bar{f}_{e}:= & \bar{\nu} u-\nu_{k} \bar{u}-r\left(\bar{u} v+u_{k} \bar{v}\right) \\
& +\bar{u}_{x} \Phi_{x}+\bar{u}(u-v)+u_{k x} \bar{\Phi}_{x}+u_{k}(\bar{u}-\bar{v}) \quad \text { in } P(T), \\
\left.\partial_{\nu} \bar{u}\right|_{\Sigma}= & \left.\left(u \partial_{\nu} \Phi-u_{k} \partial_{\nu} \Phi_{k}\right)\right|_{\Sigma} .
\end{aligned}
$$

LEMma 11. $\|\bar{u}\|_{L^{2}\left(0, T, H^{1}(\Omega)\right)} \leq C\|\bar{\sigma}\|_{\mathcal{C}^{0}}$ and $\|\bar{v}\|_{L^{2}\left(0, T, H^{1}(\Omega)\right)} \leq C\|\bar{\sigma}\|_{\mathcal{C}^{0}}$.

Lemma 12. The estimations of Lemma 11 also hold for $\left\|\bar{u}_{t}\right\|_{L^{2}\left(0, T, H^{1}(\Omega)\right)}$ and $\left\|\bar{v}_{t}\right\|_{L^{2}\left(0, T, H^{1}(\Omega)\right)}$.

LEMma 13. The estimations of Lemma 11 also hold for $\left\|\bar{u}_{t t}\right\|_{L^{2}\left(0, T, H^{1}(\Omega)\right)}$ and $\left\|\bar{v}_{t t}\right\|_{L^{2}\left(0, T, H^{1}(\Omega)\right)}$.

The proofs of Lemmas 11,12 , and 13 use the assumptions $(H)$ on the ionization function $\nu$. For details of them, we refer to [19]. 
Since $\overline{\mathcal{F}}(t, 0)=\bar{v}_{x}(t, 0)$ and

$$
\|\overline{\mathcal{F}}(s, 0)\|_{\mathcal{C}^{1}[0, t]} \leq C\left\|\bar{f}_{i}\right\|_{H^{\frac{11}{4}}, \frac{11}{8}(P(t))},
$$

estimations on $\left\|\bar{f}_{i}\right\|_{H^{\frac{11}{4}, \frac{11}{8}(P(t))}}$ are required for proving the continuity of the map $\mathcal{T}_{1}$.

(i) This step aims at showing that

$$
\left\|\bar{f}_{e}\right\|_{H^{\frac{11}{4}, \frac{11}{8}(P(t))}}^{2} \leq C\left(\|\bar{\sigma}\|_{\mathcal{C}^{\prime}}^{2}+\|\bar{u}\|_{H^{\frac{15}{4}, \frac{15}{8}(P(t))}}^{2}+\|\bar{v}\|_{H^{\frac{15}{4}, \frac{15}{8}(P(t))}}^{2}\right) .
$$

Similar inequalities also hold for $f_{i}$. Recall that

$$
u, v, u_{k}, v_{k} \in H^{\frac{19}{4}, \frac{19}{8}}(P(t)), \quad \bar{u}, \bar{v} \in H^{\frac{15}{4}, \frac{15}{8}}(P(t)) .
$$

In the following, the references to "the space part" and "the time part" of $\left\|f_{e}\right\|_{H^{r, s}}$ mean the search for bounds from above of $\left\|f_{e}\right\|_{L^{2}\left(0, T ; H^{r}(\Omega)\right)}$ and $\left\|f_{e}\right\|_{H^{s}\left(0, T ; L^{2}(\Omega)\right)}$, respectively.

For the space part, bounding from above terms like $\bar{\nu}_{u}, \bar{u} v, \bar{v}_{x} \Phi_{x}$, and $v_{x} \bar{\Phi}_{x}$ in $L^{2}(0, t$; $\left.H^{\frac{11}{4}}(\Omega)\right)$ provides a bound for $\left\|\bar{f}_{i}\right\|_{L^{2}\left(0, t ; H^{\frac{11}{4}}(\Omega)\right)}$.

- Consider $\|\bar{\nu} u\|_{L^{2}\left(H^{\frac{11}{4}}\right)}$. First,

$$
\|\bar{\nu} u\|_{L^{2}\left(H^{2}\right)}^{2}=\|\bar{\nu} u\|_{L^{2}(P(t))}^{2}+\left\|\bar{\nu}_{x x} u+2 \bar{\nu}_{x} u_{x}+\bar{\nu} u_{x x}\right\|_{L^{2}(P(t))}^{2} .
$$

By the hypothesis (H2),

$$
\left|\bar{\nu}_{x x}(t, x)\right| \leq c\left(\left|\bar{E}_{x x}\right|(t, x)+\|\bar{\sigma}\|_{\mathcal{C}^{0}}\right) .
$$

Moreover, $u$ is bounded in $\mathcal{C}^{0}(P(t))$, and so

$$
\left\|\bar{\nu}_{x x} u\right\|_{L^{2}(P(t))}^{2} \leq C\left(\|\bar{\sigma}\|_{\mathcal{C}^{0}}^{2}+\|\bar{u}(s, \cdot)-\bar{v}(s, \cdot)\|_{L^{2}\left(0, t ; H^{\frac{15}{4}}(\Omega)\right)}^{2}\right) .
$$

Analogously, by the belongness of $u$ to $H^{\frac{19}{4}, \frac{19}{8}}, u_{x}$ and $u_{x x}$ belong to $\mathcal{C}^{0}(P(t))$, so that

$$
\begin{aligned}
\left\|\bar{\nu}_{x} u_{x}\right\|_{L^{2}(P(t))}^{2} \leq C\left(\|\bar{\sigma}\|_{\mathcal{C}^{0}}^{2}+\|\bar{u}(s, \cdot)-\bar{v}(s, \cdot)\|_{L^{2}\left(0, t ; H^{\frac{15}{4}}(\Omega)\right)}^{2}\right), \\
\left\|\bar{\nu} u_{x x}\right\|_{L^{2}(P(t))}^{2} \leq C\left(\|\bar{\sigma}\|_{\mathcal{C}^{0}}^{2}+\|\bar{u}(s, \cdot)-\bar{v}(s, \cdot)\|_{L^{2}\left(0, t ; H^{\frac{15}{4}}(\Omega)\right)}^{2}\right),
\end{aligned}
$$

and

$$
\|\bar{\nu} u\|_{L^{2}\left(H^{2}\right)}^{2} \leq C\left(\|\bar{\sigma}\|_{\mathcal{C}^{0}}^{2}+\|\bar{u}\|_{L^{2}\left(0, t, H^{\frac{15}{4}}(\Omega)\right)}^{2}+\|\bar{v}\|_{L^{2}\left(0, t, H^{\frac{15}{4}}(\Omega)\right)}^{2}\right) .
$$

Then it remains to bound from above $I(t):=\left\|\bar{\nu}_{x x}(t, \cdot) u(t, \cdot)\right\|_{H^{\alpha}}^{2}$ with $\alpha:=\frac{3}{4}$.

$$
I(t) \leq \int_{\Omega} \int_{\Omega} \frac{\left|\left(\bar{\nu}_{x x}(t, x)-\bar{\nu}_{x x}(t, y)\right) u(t, x)\right|^{2}+\left|\bar{\nu}_{x x}(t, y)(u(t, x)-u(t, y))\right|^{2}}{|x-y|^{2 \alpha+1}} d y d x
$$

By the hypothesis (H2),

$$
\begin{aligned}
I(t) \leq & C \int_{\Omega} \int_{\Omega} \frac{|| \bar{E}_{x x}(t, x)-\bar{E}_{x x}(t, y)|+| x-y\left|\|\bar{\sigma}\|_{\mathcal{C}^{\circ}}\right|^{2}|u(t, x)|^{2}}{|x-y|^{2 \alpha+1}} d x d y \\
& +\int_{\Omega} \int_{\Omega} \frac{\left|\bar{\nu}_{x x}(t, y)(u(t, x)-u(t, y))\right|^{2}}{|x-y|^{2 \alpha+1}} d x d y .
\end{aligned}
$$


Then $\left|\bar{E}_{x x}(\tau, x)-\bar{E}_{x x}(\tau, y)\right| \leq C\|(\bar{u}-\bar{v})(\tau, \cdot)\|_{H^{\frac{15}{4}}(\Omega)}|x-y|$. Hence, by the belongness of $u$ to $\mathcal{C}^{0}(P(t))$,

$$
\begin{aligned}
I(t) \leq & C \int_{\Omega} \int_{\Omega}|x-y|^{2} \frac{\|\bar{\sigma}\|_{\mathcal{C}^{0}}^{2}+\|\bar{u}-\bar{v}\|_{H^{\frac{15}{4}}(\Omega)}^{2}}{|x-y|^{2 \alpha+1}} d x d y \\
& +\int_{\Omega} \int_{\Omega} \frac{\left|\bar{\nu}_{x x}(t, y)(u(t, x)-u(t, y))\right|^{2}}{|x-y|^{2 \alpha+1}} d x d y .
\end{aligned}
$$

Consequently, by (H1),

$$
I(t) \leq C\left(\iint \frac{d \rho d \theta}{\rho^{2 \alpha-2}}+\|u(t, \cdot)\|_{H^{\frac{3}{4}}}^{2}\right)\left(\|\bar{\sigma}\|_{\mathcal{C}^{0}}^{2}+\|\bar{u}-\bar{v}\|_{H^{\frac{15}{4}}(\Omega)}^{2}\right) .
$$

It follows that

$$
\left\|\bar{\nu}_{x x} u\right\|_{L^{2}\left(H^{\frac{3}{4}}\right)}^{2} \leq C\left(\|\bar{\sigma}\|_{\mathcal{C}^{0}}^{2}+\|\bar{u}-\bar{v}\|_{L^{2}\left(H^{\frac{15}{4}}\right)}^{2}\right) .
$$

The other terms can be treated in the same way, so that

$$
\|\bar{\nu} u\|_{L^{2}\left(0, t ; H^{\frac{11}{4}}(\Omega)\right)} \leq C\left(\|\bar{\sigma}\|_{\mathcal{C}^{0}}^{2}+\|\bar{u}-\bar{v}\|_{L^{2}\left(0, t, H^{\frac{15}{4}}(\Omega)\right)}^{2}\right) .
$$

- Consider $\|\bar{u} v\|_{L^{2}\left(H^{\frac{11}{4}}\right)}$. Since $\frac{11}{4}>\frac{1}{2}, H^{\frac{11}{4}}(\Omega)$ is a Banach algebra. Moreover, $v \in H^{\frac{19}{4}, \frac{19}{8}}$, hence $v \in \mathcal{C}^{0}\left(H^{\frac{15}{4}}\right)$. Consequently,

$$
\|\bar{u}(s, \cdot) v(s, \cdot)\|_{H^{\frac{11}{4}}} \leq C\|\bar{u}(s, \cdot)\|_{H^{\frac{15}{4}}}\|v\|_{L^{\infty}\left(H^{\frac{11}{4}}\right)},
$$

and so

$$
\|\bar{u} v\|_{L^{2}\left(H^{\frac{11}{4}}\right)} \leq C\|\bar{u}\|_{L^{2}\left(H^{\frac{15}{4}}\right)}\|v\|_{L^{\infty}\left(H^{\frac{11}{4}}\right)}
$$

- Similarly,

$$
\begin{aligned}
& \left\|\bar{v}_{x} \Phi_{x}\right\|_{L^{2}\left(H^{\frac{11}{4}}\right)} \leq C\|\bar{v}\|_{L^{2}\left(H^{\frac{15}{4}}\right)}\|u-v\|_{L^{\infty}\left(H^{\frac{7}{4}}\right)}, \\
& \left\|v_{x} \bar{\Phi}_{x}\right\|_{L^{2}\left(H^{\frac{11}{4}}\right)} \leq C\|\bar{u}\|_{L^{2}\left(H^{\frac{7}{4}}\right)}\|v\|_{L^{\infty}\left(H^{\frac{15}{4}}\right)} .
\end{aligned}
$$

For the time part, for proving that $\|\bar{f}\|_{H^{\frac{11}{8}}\left(0, t ; L^{2}(\Omega)\right)}$ is bounded from above, it is enough to prove that $\|\bar{f}\|_{H^{2}\left(0, t ; L^{2}(\Omega)\right)}$ is bounded from above.

- Consider first $\|\bar{\nu} u\|_{H^{2}\left(L^{2}\right)}$. By Lemma 13,

$$
\left\|\bar{\nu}_{t t} u\right\|_{L^{2}(P(t))}^{2} \leq C \int_{P(t)}\left(\bar{\Phi}_{t t x}+\|\bar{\sigma}\|_{\mathcal{C}^{0}}\right)^{2} u^{2} \leq C\|\bar{\sigma}\|_{\mathcal{C}^{0}}^{2},
$$

because $u$ is uniformly bounded in $L^{\infty}(P(t))$. Then

$$
\begin{aligned}
\left\|\bar{\nu} u_{t t}\right\|_{L^{2}(P(t))}^{2} & \leq C \int_{P(t)}\left(\bar{\Phi}_{x}+\|\bar{\sigma}\|_{\mathcal{C}^{0}}\right)^{2} u_{t t}^{2} \\
& \leq C \int_{0}^{t}\left\|u_{t t}(s, \cdot)\right\|_{L^{2}(\Omega)}^{2}\left(\|\bar{u}(s, \cdot)-\bar{v}(s, \cdot)\|_{L^{2}(\Omega)}^{2}+\|\bar{\sigma}\|_{\mathcal{C}^{0}}^{2}\right) \\
& \leq C\|\bar{\sigma}\|_{\mathcal{C}^{0}}^{2},
\end{aligned}
$$

thanks to Lemma 11 and the boundedness of $u_{t t}$ in $L^{\infty}\left(L^{2}\right)$. 
- Let us bound from above $\|\nu \bar{u}\|_{H^{2}\left(L^{2}\right)}$. Thanks to Lemmas 7 and 11,

$$
\left\|\nu_{t t} \bar{u}\right\|_{L^{2}(P(t))}^{2} \leq C \int_{P(t)}\left(\Phi_{t t x}+\|\sigma\|_{\mathcal{C}^{0}}\right)^{2} \bar{u}^{2} \leq C\|\bar{\sigma}\|_{\mathcal{C}^{0}}^{2} .
$$

Then

$$
\left\|\nu \bar{u}_{t t}\right\|_{L^{2}(P(t))}^{2} \leq C \int_{P(t)}\left(\Phi_{x}+\|\bar{\sigma}\|_{\mathcal{C}^{0}}\right)^{2} \bar{u}_{t t}^{2} \leq C\|\bar{\sigma}\|_{\mathcal{C}^{0}}^{2}
$$

as previously.

- Let us bound from above $\|\bar{u} v\|_{H^{2}\left(L^{2}\right)}$. Since $v$ is uniformly bounded, $\left\|\bar{u}_{t t} v\right\|_{L^{2}(P(t))}^{2} \leq$ $c\|\bar{\sigma}\|_{\mathcal{C}^{0}}^{2}$.

Then $\left\|\bar{u} v_{t t}\right\|_{L^{2}(P(t))}^{2} \leq c \int_{0}^{t}\|\bar{u}\|_{H^{1}(\Omega)}^{2}\left\|v_{t t}\right\|_{L^{2}(\Omega)}^{2} \leq c\|\bar{\sigma}\|_{\mathcal{C}^{0}}^{2}$, thanks to Lemmas 7 and 11 .

- Consider $\left\|\bar{v}_{x} \Phi_{x}\right\|_{H^{2}\left(L^{2}\right)}$.

$$
\begin{aligned}
\left\|\bar{v}_{t t x} \Phi_{x}\right\|_{L^{2}(P(t))}^{2} & \leq C \int_{0}^{t}\left(\|u-v\|_{L^{2}(\Omega)}+\|g\|_{H^{\frac{1}{2}}(\Gamma)}\right)^{2}\left\|\bar{v}_{t t}\right\|_{H^{1}(\Omega)}^{2} \\
& \leq C \int_{0}^{t}\left\|\bar{v}_{t t}\right\|_{H^{1}(\Omega)}^{2} \\
& \leq C\|\bar{\sigma}\|_{\mathcal{C}^{0}} .
\end{aligned}
$$

- Consider $\left\|v_{x} \bar{\Phi}_{x}\right\|_{H^{2}\left(L^{2}\right)}$. Since $\bar{u}$ and $\bar{v}$ belong to $H^{\frac{15}{4}, \frac{15}{8}}, \bar{u}$ and $\bar{v}$ belong to $\mathcal{C}^{0}\left(L^{2}(\Omega)\right)$, so that

$$
\begin{aligned}
& \left\|v_{t t x} \bar{\Phi}_{x}\right\|_{L^{2}(P(t))}^{2} \leq C \int_{0}^{t}\left(\|\bar{u}-\bar{v}\|_{L^{2}(\Omega)}\right)^{2}\left\|v_{t t}\right\|_{H^{1}(\Omega)}^{2} \\
& \leq C\left(\sup _{s \in(0, t)}\|\bar{u}(s, \cdot)-\bar{v}(s, \cdot)\|_{L^{2}(\Omega)}^{2}\right)\left\|v_{t t}\right\|_{L^{2}\left(H^{1}\right)}^{2}
\end{aligned}
$$

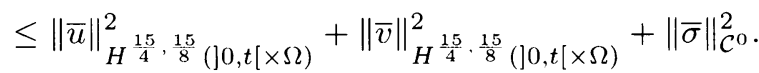

Then, since $v \in \mathcal{C}^{0}\left(H^{1}(\Omega)\right)$,

$$
\left\|v_{x} \bar{\Phi}_{t t x}\right\|_{L^{2}(P(t))}^{2} \leq C \int_{0}^{t}\left(\left\|\bar{u}_{t t}-\bar{v}_{t t}\right\|_{L^{2}(\Omega)}\right)^{2}\|v\|_{H^{1}}^{2} \leq C\|\bar{\sigma}\|_{\mathcal{C}^{0}}^{2} .
$$

It follows from this first step that

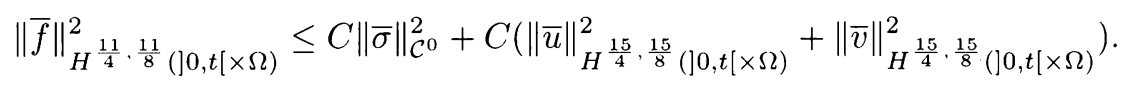

(ii) By Lions-Magenes [12],

$$
\begin{aligned}
\|\bar{u}\|_{H^{\frac{15}{4}, \frac{15}{8}}(P(t))} \leq C\left(\left\|\bar{f}_{e}\right\|_{H^{\frac{7}{4}, \frac{7}{8}(P(t))}}+\left\|\bar{g}_{e}\right\|_{H^{\frac{9}{8}\left(\Sigma_{t}\right)}}\right), \\
\|\bar{v}\|_{H^{\frac{15}{4}, \frac{15}{8}(P(t))}} \leq C\left\|\bar{f}_{i}\right\|_{H^{\frac{7}{4}, \frac{7}{8}(P(t))}} .
\end{aligned}
$$

(iii) The following inequalities hold:

$$
\begin{aligned}
\left\|\bar{f}_{e}\right\|_{H^{\frac{7}{4}, \frac{7}{8}(P(t))}}^{2}+\left\|\bar{f}_{i}\right\|_{H^{\frac{7}{4}, \frac{7}{8}(P(t))}}^{2} & \leq C\left(\|\bar{\sigma}\|_{\mathcal{C}^{0}}^{2}+\|\bar{u}\|_{H^{\frac{11}{4}, \frac{11}{8}}(P(t))}^{2}+\|\bar{v}\|_{H^{\frac{11}{4}, \frac{11}{8}(P(t))}}^{2}\right), \\
\left\|\bar{g}_{e}\right\|_{H^{\frac{9}{8}(P(t))}}^{2} & \leq C\left(\|\bar{u}\|_{H^{\frac{11}{4}, \frac{11}{8}(P(t))}}^{2}+\|\bar{v}\|_{H^{\frac{11}{4}, \frac{11}{8}(P(t))}}^{2}\right) .
\end{aligned}
$$


Indeed, such estimations on $\left\|\bar{f}_{e}\right\|_{H^{\frac{7}{4}, \frac{7}{8}(P(t))}}$ and $\left\|\bar{f}_{i}\right\|_{H^{\frac{7}{4}, \frac{7}{8}(P(t))}}$ can be handled as in Step 1. Then

$$
\left\|\bar{g}_{e}\right\|_{H^{\frac{9}{8}}} \leq C\left\|\bar{u} \Phi_{x}+u \bar{\Phi}_{x}\right\|_{H^{\frac{11}{4}, \frac{11}{8}(P(t))}} .
$$

For the space part, the belongness of $u, u_{x}, u_{x x}$ to $\mathcal{C}^{0}\left(L^{2}\right)$ implies that

$$
\begin{aligned}
& \iint \bar{u}_{x x}^{2} \Phi_{x}^{2} \leq C \int_{0}^{t}\|u(s, \cdot)\|_{L^{2}}^{2}\left\|\bar{u}_{x x}(s, \cdot)\right\|_{L^{2}}^{2} \leq C\|\bar{u}\|_{L^{2}\left(H^{2}\right)}^{2}, \\
& \iint \bar{u}^{2} \Phi_{x x x}^{2} \leq C \int_{0}^{t}\left\|u_{x}\right\|_{L^{2}}^{2}\|\bar{u}\|_{L^{2}}^{2} \leq C\|\bar{u}\|_{L^{2}}^{2} .
\end{aligned}
$$

Moreover,

$$
\begin{aligned}
& \iint u_{x x}^{2} \bar{\Phi}_{x}^{2} \leq C\|\bar{u}\|_{L^{2}}^{2} \\
& \iint u^{2} \bar{\Phi}_{x x x}^{2} \leq C\|\bar{u}\|_{L^{2}\left(H^{2}\right)}^{2}
\end{aligned}
$$

Hence,

$$
\left\|\bar{u} \Phi_{x}+u \bar{\Phi}_{x}\right\|_{L^{2}\left(H^{\frac{11}{4}}\right)}^{2} \leq C\|\bar{u}\|_{L^{2}\left(H^{\frac{11}{4}}\right)}^{2}+C\|\bar{v}\|_{L^{2}\left(H^{\frac{11}{4}}\right)}^{2} .
$$

For the time part,

$$
\begin{aligned}
\left\|\bar{u} \Phi_{x}+u \bar{\Phi}_{x}\right\|_{H^{2}\left(L^{2}\right)}^{2}= & \left\|\bar{u} \Phi_{x}+u \bar{\Phi}_{x}\right\|_{L^{2}(P(t))}^{2} \\
& +\left\|\bar{u}_{t t} \Phi_{x}+2 \bar{u}_{t} \Phi_{t x}+\bar{u} \Phi_{t t x}\right\|_{L^{2}(P(t))}^{2} \\
& +\left\|u_{t t} \bar{\Phi}_{x}+2 u_{t} \bar{\Phi}_{t x}+u \bar{\Phi}_{t t x}\right\|_{L^{2}(P(t))}^{2}
\end{aligned}
$$

It is easy to see that

$$
\begin{aligned}
\left\|\bar{u}_{t t} \Phi_{x}\right\|_{L^{2}(P(t))}^{2} & \leq C\|\bar{\sigma}\|_{\mathcal{C}^{0}}^{2}, \\
\left\|\bar{u} \Phi_{t t x}\right\|_{L^{2}(P(t))}^{2} & \leq C \int_{0}^{t}\left(\left\|u_{t t}-v_{t t}\right\|_{L^{2}(\Omega)}+\left\|g_{t t}\right\|_{H^{1}}\right)^{2}\|\bar{u}\|_{L^{2}(\Omega)}^{2} \\
& \leq C\|\bar{\sigma}\|_{\mathcal{C}^{0}}^{2},
\end{aligned}
$$

and the other terms are treated similarly. Hence,

$$
\left\|\bar{g}_{e}\right\|_{H^{\frac{9}{8}\left(\Sigma_{t}\right)}}^{2} \leq C\left(\|\bar{u}\|_{H^{\frac{11}{4}, \frac{11}{8}(P(t))}}^{2}+\|\bar{v}\|_{H^{\frac{11}{4}, \frac{11}{8}(P(t))}}^{2}\right) .
$$

(iv) As previously,

$$
\begin{aligned}
&\|\bar{u}\|_{H^{\frac{11}{4}, \frac{11}{8}(P(t))}}^{2} \leq C\left(\left\|\bar{f}_{e}\right\|_{H^{\frac{3}{4}, \frac{3}{8}(P(t))}}^{2}+\left\|\bar{g}_{e}\right\|_{H^{\frac{5}{8}\left(\Sigma_{t}\right)}}^{2}\right), \\
&\|\bar{v}\|_{H^{\frac{11}{4}, \frac{11}{8}(P(t))}}^{2} \leq C\left\|\bar{f}_{i}\right\|_{H^{\frac{3}{4}, \frac{3}{8}(P(t))}}^{2} .
\end{aligned}
$$

(v) By a similar computation to Step 1,

$$
\left\|\bar{f}_{e}\right\|_{H^{\frac{3}{4}, \frac{3}{8}(P(t))}}^{2} \leq C\left(\|\bar{\sigma}\|_{\mathcal{C}^{0}}^{2}+\|\bar{u}\|_{H^{\frac{7}{4}, \frac{7}{8}((0, t) \times \Omega)}}^{2}+\|\bar{v}\|_{H^{\frac{7}{4}, \frac{7}{8}}((0, t) \times \Omega)}^{2}\right) .
$$


(vi) Again,

$$
\begin{aligned}
& \|\bar{u}\|_{H^{\frac{7}{4}, \frac{7}{8}(P(t))}}^{2} \leq C\left(\left\|\bar{f}_{e}\right\|_{L^{2}(P(t))}^{2}+\left\|\bar{g}_{e}\right\|_{H^{\frac{1}{4}}\left(\Sigma_{t}\right)}^{2}\right), \\
& \|\bar{v}\|_{H^{\frac{7}{4}, \frac{7}{8}(P(t))}}^{2} \leq C\left\|\bar{f}_{i}\right\|_{L^{2}(P(t))}^{2} .
\end{aligned}
$$

(vii) It is easy to see that

$$
\begin{aligned}
\left\|\bar{f}_{e}\right\|_{L^{2}(P(t))} & \leq C\left(\|\bar{\sigma}\|_{\mathcal{C}^{0}}^{2}+\|\bar{u}\|_{L^{2}\left(H^{1}\right)}^{2}+\|\bar{v}\|_{L^{2}\left(H^{1}\right)}^{2}\right) \\
\left\|\bar{g}_{e}\right\|_{H^{\frac{1}{4}\left(\Sigma_{t}\right)}}^{2} & \leq C\left(\|\bar{u}\|_{H^{1, \frac{1}{2}}}^{2}+\|\bar{v}\|_{H^{1, \frac{1}{2}}}^{2}\right) .
\end{aligned}
$$

(viii) Moreover,

$$
\begin{aligned}
\|\bar{u}\|_{H^{1, \frac{1}{2}}}^{2} & \leq C\left(\|\bar{u}\|_{L^{2}\left(H^{1}\right)}^{2}+\left\|\bar{u}_{t}\right\|_{L^{2}\left(H^{1}\right)}^{2}\right), \\
\|\bar{v}\|_{H^{1, \frac{1}{2}}}^{2} & \leq C\left(\|\bar{v}\|_{L^{2}\left(H^{1}\right)}^{2}+\left\|\bar{v}_{t}\right\|_{L^{2}\left(H^{1}\right)}^{2}\right) .
\end{aligned}
$$

(ix) Lemmas 11 and 12 imply that $\|\bar{u}\|_{L^{2}\left(0, T, H^{1}(\Omega)\right)} \leq C\|\bar{\sigma}\|_{\mathcal{C}^{0}}$ and $\left\|\bar{u}_{t}\right\|_{L^{2}\left(0, T, H^{1}(\Omega)\right)} \leq$ $C(\bar{\sigma})_{\mathcal{C}^{0}}$. So $\|\bar{u}\|_{H^{1, \frac{1}{2}}}^{2} \leq C\|\bar{\sigma}\|_{\mathcal{C}^{0}}$. The same result holds for $\|\bar{v}\|_{H^{1, \frac{1}{2}}}^{2}$, and so, it has been proven that

$$
\|\overline{\mathcal{F}}(s, \cdot)\|_{\mathcal{C}^{1}([0, t])} \leq C\|\overline{\mathcal{F}}(s, \cdot)\|_{H^{\frac{13}{8}}(0, t)} \leq C\|\bar{\sigma}\|_{\mathcal{C}^{0}}
$$

This proves the continuity of the map $\mathcal{T}_{1}$, which ends the proof of Theorem 10 .

4.2. Continuity of the map $\mathcal{T}_{2}$. By Corollary $9, \mathcal{F}(t) \in \mathcal{C}^{1}([0, T])$. Let $\mathcal{T}_{2}$ be the following map:

$$
\begin{aligned}
C^{1}([0, \delta]) & \rightarrow \Sigma(P(\delta)), \\
\mathcal{F} & \mapsto \rho,
\end{aligned}
$$

where $\rho$ is the first component of the solution $(\rho, m)$ to

$$
\left\{\begin{array}{l}
-\lambda_{2}\left(\rho_{t}+\lambda_{1} \rho_{x}\right)+m_{t}+\lambda_{1} m_{x}=0 \quad \text { in } P(\delta), \\
-\lambda_{1}\left(\rho_{t}+\lambda_{2} \rho_{x}\right)+m_{t}+\lambda_{2} m_{x}=0 \quad \text { in } P(\delta), \\
\rho(0, x)=\rho_{0}(x), \quad m(0, x)=m_{0}(x) \quad \text { in } \Omega
\end{array}\right.
$$

together with the boundary conditions

$$
\begin{aligned}
m(t, 0) & =\mathcal{F}(t), \\
\rho(t, a) & =\rho_{a}(t) .
\end{aligned}
$$

The continuity of $\mathcal{T}_{2}$ is proven in $\mathcal{C}^{0}(P(\delta))$. In fact, it is sufficient to study it in $\mathcal{C}^{0}(R(\delta) \cup$ $S(\delta)$ ) since the solution on the domain $Q(\delta)$ does not depend on the boundary conditions $m$ on $x=0$ and $\rho$ on $x=a$. The computations being the same on $R(\delta)$ and $S(\delta)$, restrict to $\Sigma(R(\delta))$. 
4.3. The boundary value problem on the domain $R(\delta)$. Denote by $\omega^{1}:=\rho$ and $\omega^{2}:=$ $m$. The following problem is equivalent to the system (1.2) (see [19]):

$$
\left\{\begin{array}{l}
\sum_{j=1}^{2} \zeta_{l j}(t, x, \omega)\left(\partial_{t} \omega_{j}+\lambda_{l}(t, x, \omega) \partial_{x} \omega_{j}\right)=0, \quad l=1,2, \quad(t, x) \in R(\delta), \\
\sum_{j=1}^{2} \zeta_{1 j}^{0} \omega_{j}=G_{1}(t):=-\lambda_{2}^{0}\left(\rho^{c}-\tilde{\rho}^{0}\right)+\left(m^{c}-\tilde{m}^{0}\right), \quad x=x_{1}(t), \\
\sum_{j=1}^{2} \zeta_{2 j}^{0} \omega_{j}=G_{2}(t, \omega)-\lambda_{1}^{0}\left(\rho-\tilde{\rho}^{0}\right)+\left(\mathcal{F}-\tilde{m}^{0}\right), \quad x=0
\end{array}\right.
$$

where the matrix $\left(\zeta_{l j}\right)_{l, j=1,2}$ is given by $\left(\zeta_{l j}\right)=\left(\begin{array}{ll}-\lambda_{2} & 1 \\ -\lambda_{1} & 1\end{array}\right)$.

By Theorem 9 , the function $\mathcal{F}$ belongs to $C^{1}([0, T])$. It follows from the first inequality of (4.13) that the minimal characterizing number $\theta_{\min }$ is smaller than one. As in Sec. 3 , there exists a solution to (4.27) that belongs to $\mathcal{C}^{1}(R(\delta))$ for $\delta$ small enough. The continuity of the map

$$
\mathcal{F} \rightarrow \omega:=(\rho, m)
$$

where $(\rho, m)$ is the solution to $(4.27)$ is proven in the following way. Let $\left(\mathcal{F}^{p}\right)$ be a sequence converging in $\mathcal{C}^{1}([0, T])$ to $\mathcal{F}$. Let $\omega$ and $\omega^{p}$ be solutions to (4.27), associated to $\mathcal{F}$ and $\mathcal{F}^{p}$ respectively. The difference $\bar{\omega}:=\omega-\omega^{p}$ satisfies

$$
\begin{aligned}
& \sum_{j=1}^{2} \zeta_{l j}(t, x, \omega)\left(\partial_{t} \bar{\omega}_{j}+\lambda_{l}(t, x, \omega) \partial_{x} \bar{\omega}_{j}\right) \\
& =\sum_{j=1}^{2} \partial_{t} \omega_{j}^{p}\left(\zeta_{l j}\left(t, x, \omega^{p}\right)-\zeta_{l j}(t, x, \omega)\right) \\
& \quad+\sum_{j=1}^{2} \partial_{x} \omega_{j}^{p}\left(\zeta_{l j}\left(t, x, \omega^{p}\right) \lambda_{l}\left(t, x, \omega^{p}\right)-\zeta_{l j}(t, x, \omega) \lambda_{l}(t, x, \omega)\right) \\
& =: \quad h_{l}\left(\omega, \omega^{p}\right), \quad l=1,2 .
\end{aligned}
$$

The $l$-characteristics $f_{l}(\tau ; t, x)$ starting at $(t, x)$ are defined by

$$
\left\{\begin{array}{l}
\frac{d f_{l}(\tau ; t, x)}{d \tau}=\lambda_{l}\left(\tau, f_{l}, \omega\left(\tau, f_{l}\right)\right) \\
f_{l}(t ; t, x)=x, \quad l \in\{1,2\}
\end{array}\right.
$$

Let $\tau_{1}$ and $\tau_{2}$ be the larger times such that

$$
f_{2}\left(\tau_{2}(t, x) ; t, x\right)=0 \quad \text { and } \quad f_{1}\left(\tau_{1}(t, x) ; t, x\right)=x_{1}\left(\tau_{1}\right)
$$


Integrating (4.28) on the $l$-characteristics between $\tau_{l}$ and $t$ and multiplying by the inverse matrix $\left(\zeta^{k l}\right)$ of $\left(\zeta_{l j}\right)$ imply that

$$
\begin{aligned}
\bar{\omega}_{k}(t, x)= & \sum_{l, j=1}^{2} \zeta^{k l}(t, x, \omega) \zeta_{l j}\left(\tau_{l}, f_{l}\left(\tau_{l}\right), \omega\left(\tau_{l}, f_{l}\left(\tau_{l}\right)\right)\right) \bar{\omega}_{j}\left(\tau_{l}, f_{l}\left(\tau_{l}\right)\right) \\
& +\sum_{l} \zeta^{k l}(t, x, \omega) \sum_{j=1}^{2} \int_{\tau_{l}}^{t} \frac{d \zeta_{l j}(\omega)}{d_{l} \tau} \bar{\omega}_{j} d \tau+\sum_{l} \zeta^{k l}(t, x, u) \int_{\tau_{l}}^{t} h_{l}\left(u, u_{p}\right) d \tau,
\end{aligned}
$$

where for any function $K(t, x)$,

$$
\frac{d K\left(\tau, f_{l}(\tau)\right)}{d_{l} \tau}:=\partial_{t} K\left(\tau, f_{l}(\tau)\right)+\lambda_{l} \partial_{x} K\left(\tau, f_{l}(\tau)\right), \quad l \in\{1,2\}
$$

It follows from $\bar{\omega}=0$ at the points $\left(t, x_{1}(t)\right)$ that

$$
\begin{aligned}
\bar{\omega}_{k}(t, x)= & \zeta^{k 2}(t, x, \omega)\left(-\lambda_{1}\left(\tau_{2}, 0, \omega\left(\tau_{2}, 0\right)\right) \bar{\omega}_{1}\left(\tau_{2}, 0\right)+\bar{\omega}_{2}\left(\tau_{2}, 0\right)\right) \\
& +\sum_{l} \zeta^{k l}(t, x, \omega) \sum_{j=1}^{2} \int_{\tau_{l}}^{t} \frac{d \zeta_{l j}(\omega)}{d_{l} \tau} \bar{\omega}_{j} d \tau+\sum_{l} \zeta^{k l}(t, x, \omega) \int_{\tau_{l}}^{t} h_{l}\left(\omega, \omega_{p}\right) d \tau .
\end{aligned}
$$

Only $\bar{\omega}_{2}\left(\tau_{2}, 0\right)$ is given by the boundary condition. In order to know $\bar{\omega}_{1}\left(\tau_{2}, 0\right)$, an integration of (4.28) on the first characteristics between $\tau_{1}\left(\tau_{2}(t, x)\right)$ and $\tau_{2}(t, x)$ is performed. Hence,

$$
\zeta_{11}\left(\tau_{2}, 0, \omega\left(\tau_{2}, 0\right)\right) \bar{\omega}_{1}\left(\tau_{2}, 0\right)=-\bar{\omega}_{2}\left(\tau_{2}, 0\right)+\sum_{j=1}^{2} \int_{\tau_{1}}^{\tau_{2}} \frac{d \zeta_{1 j}(\omega)}{d_{1} \tau} \bar{\omega}_{j} d \tau+\int_{\tau_{1}}^{\tau_{2}} h_{1}\left(\omega, \omega^{p}\right) d \tau
$$

since $\bar{\omega}=0$ on $\left(t, x_{1}(t)\right)$ and $\zeta_{12}=1$. Since $\omega$ belongs to $\mathcal{C}^{1}, \zeta_{11}\left(\tau_{2}, 0, \omega\left(\tau_{2}, 0\right)\right)$ is bounded. Hence

$$
\begin{aligned}
\left|\bar{\omega}_{k}(t, x)\right| & \leq C\left(\left|\bar{\omega}_{2}\left(\tau_{2}, 0\right)\right|+\sum_{l} \sum_{j=1}^{2} \int_{\tau_{l}}^{t}\left|\bar{\omega}_{j}\right| d \tau+\sum_{l} \int_{\tau_{l}}^{t}\left|h_{l}\left(\omega, \omega_{p}\right)\right| d \tau\right) \\
& \leq C\left(\left|\bar{\omega}_{2}\left(\tau_{2}, 0\right)\right|+\sum_{j=1}^{2} \int_{0}^{t}\left|\bar{\omega}_{j}\left(\tau, f_{l}(\tau)\right)\right| d \tau\right) .
\end{aligned}
$$

Let

$$
\mathcal{V}(t):=\sup _{\substack{j \\ s, x \in R(t)}}\left|\bar{\omega}_{j}(s, x)\right|, \quad t \in[0, \delta],
$$

where $R(t)$ is defined by $R(t)=\left\{(\tau, x) ; 0 \leq \tau \leq t, 0 \leq x \leq x_{1}(\tau)\right\}$. On the $f_{l}$ characteristics, $\bar{\omega}_{j}\left(\tau, f_{l}(\tau)\right) \leq \mathcal{V}(\tau)$. Hence

$$
\mathcal{V}(t) \leq C\left|\bar{\omega}_{2}\left(\tau_{2}, 0\right)\right|+C \int_{0}^{t} \mathcal{V}(\tau) d \tau
$$

It follows from Gronwall's lemma that $\mathcal{V}(t) \leq C\left\|\bar{\omega}_{2}\right\|_{C^{0}}=\|\overline{\mathcal{F}}\|_{C^{0}}$. Hence $\mathcal{V}$ tends to zero in $C^{0}$ when $\overline{\mathcal{F}}$ tends to zero. 
4.4. Uniqueness of the solution to the whole system. Let $\left(u^{1}, v^{1}, \Phi^{1}, \rho^{1}, m^{1}\right)$ and $\left(u^{2}, v^{2}, \Phi^{2}, \rho^{2}, m^{2}\right)$ be two solutions of the system (1.1)-(1.2). We denote by - all the quantities expressing differences. For example, $\bar{\omega}:=\left(\omega_{1}^{1}-\omega_{1}^{2}, \omega_{2}^{1}-\omega_{2}^{2}\right), \bar{u}:=u^{1}-u^{2}, \ldots$ Subtracting the equations satisfied by these solutions,

$$
\begin{aligned}
\partial_{t} \bar{u}-\partial_{x x} \bar{u} & =\bar{\nu} u-\nu^{2} \bar{u}-r\left(\bar{u} v^{1}+u^{2} \bar{v}\right)+\bar{u}_{x} \Phi_{x}^{1}+\bar{u}\left(u^{1}-v^{1}\right)+u_{x}^{2} \bar{\Phi}_{x}+u^{2}(\bar{u}-\bar{v}), \\
\partial_{\nu} \bar{u} & =u^{1} \Phi_{1, x}-u^{2} \Phi_{2, x}, \quad(t, x) \in \Sigma
\end{aligned}
$$

and

$$
\begin{aligned}
& \sum_{j=1}^{2} \zeta_{l j}\left(t, x, \omega^{1}\right)\left(\partial_{t} \bar{\omega}_{j}+\lambda_{l}\left(t, x, \omega^{1}\right) \partial_{x} \bar{\omega}_{j}\right) \\
& =\sum_{j=1}^{2} \partial_{t} \omega_{j}^{2}\left(\zeta_{l j}\left(t, x, \omega^{1}\right)-\zeta_{l j}\left(t, x, \omega^{2}\right)\right) \\
& \quad+\sum_{j=1}^{2} \partial_{x} \omega_{j}^{2}\left(\zeta_{l j}\left(t, x, \omega^{2}\right) \lambda_{l}\left(t, x, \omega^{2}\right)-\zeta_{l j}\left(t, x, \omega^{1}\right) \lambda_{l}\left(t, x, \omega^{1}\right)\right) \\
& :=h_{l}\left(\omega^{1}, \omega^{2}\right) \quad(l=1,2) .
\end{aligned}
$$

Let

$$
\mathcal{U}(t):=\sup _{\substack{j \\ s, x \in R(t)}}\left|\bar{\omega}_{j}(s, x)\right| .
$$

With similar computations to Subsection 4.3, i.e., using a method based on the characteristic curves, it can be proven that

$$
\mathcal{U}(t) \leq C\left\|\bar{u}_{2}\right\|_{C^{0}} \leq c\left\|\bar{v}_{x}\right\|_{\mathcal{C}^{0}} .
$$

Then, the following inequality,

$$
\left\|\bar{v}_{x}\right\|_{\mathcal{C}^{0}} \leq C \int_{0}^{t} \sup _{x} \bar{\rho}^{2}(s, \cdot) d s
$$

is proven in the following way.

LEMMA 14.

$$
\|\bar{u}\|_{L^{2}\left(0, T ; H^{1}(\Omega)\right)}^{2} \leq C \int_{0}^{t} \sup _{x}|\bar{\rho}(s, x)|^{2} d s
$$

and

$$
\|\bar{v}\|_{L^{2}\left(0, T ; H^{1}(\Omega)\right)}^{2} \leq C \int_{0}^{t} \sup _{x}|\bar{\rho}(s, x)|^{2} d s .
$$

Lemma 15. The estimations of Lemma 14 are also valid for $\left\|\bar{u}_{t}\right\|_{L^{2}\left(0, T, H^{1}(\Omega)\right)}$, $\left\|\bar{v}_{t}\right\| L^{2}\left(0, T, H^{1}(\Omega)\right),\left\|\bar{u}_{t t}\right\|_{L^{2}\left(0, T, H^{1}(\Omega)\right)}$, and $\left\|\bar{v}_{t t}\right\|_{L^{2}\left(0, T, H^{1}(\Omega)\right)}$.

Proof. The proofs of Lemma 14 and Lemma 15 are analogous to the proof of Lemma 11. For details we refer to [19]. 
Denote by

$$
\bar{f}_{i}:=\bar{\nu} u-\nu^{2} \bar{u}-r\left(\bar{u} v^{1}+u^{2} \bar{v}\right)+\bar{u}_{x} \Phi_{x}^{1}+\bar{u}\left(u^{1}-v^{1}\right)+u_{x}^{2} \bar{\Phi}_{x}+u^{2}(\bar{u}-\bar{v}) .
$$

The difference of the fluxes of ions is $\overline{\mathcal{F}}(t, \cdot)=\bar{v}_{x}(t, \cdot)$ on $\Gamma$. Then,

$$
\|\overline{\mathcal{F}}\|_{\mathcal{C}^{1}[0, t]} \leq c\|\overline{\mathcal{F}}(s, \cdot)\|_{H^{\frac{13}{8}}(0, t)} \leq C\left\|\bar{f}_{i}\right\|_{H^{\frac{11}{4}, \frac{11}{8}\left(P_{t}\right)}} .
$$

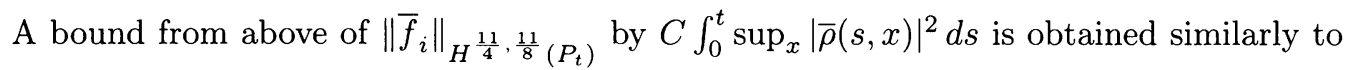
the proof of Subsection 4.1. We only give the main steps of the rest of the proof.

(i) First,

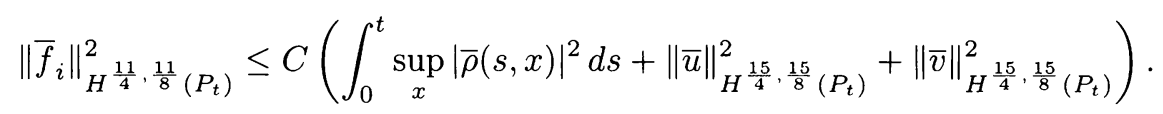

(ii) Then, by Lions-Magenes [12],

$$
\begin{aligned}
\|\bar{u}\|_{H^{\frac{15}{4}, \frac{15}{8}\left(P_{t}\right)}} \leq C\left(\left\|\bar{f}_{e}\right\|_{H^{\frac{7}{4}, \frac{7}{8}\left(P_{t}\right)}}+\left\|\bar{g}_{e}\right\|_{H^{\frac{9}{8}\left(\Sigma_{t}\right)}}\right), \\
\|\bar{v}\|_{H^{\frac{15}{4}, \frac{15}{8}\left(P_{t}\right)}} \leq C\left\|\bar{f}_{i}\right\|_{H^{\frac{7}{4}, \frac{7}{8}\left(P_{t}\right)}} .
\end{aligned}
$$

(iii) Then it is shown that

$$
\begin{aligned}
& \left\|\bar{f}_{e}\right\|_{H^{\frac{7}{4}, \frac{7}{8}\left(P_{t}\right)}}^{2} \leq C\left(\int_{0}^{t} \sup _{x}|\bar{\rho}(s, x)|^{2} d s+\|\bar{u}\|_{H^{\frac{11}{4}, \frac{11}{8}\left(P_{t}\right)}}^{2}+\|\bar{v}\|_{H^{\frac{11}{4}, \frac{11}{8}\left(P_{t}\right)}}^{2}\right), \\
& \left\|\bar{f}_{i}\right\|_{H^{\frac{7}{4}, \frac{7}{8}\left(P_{t}\right)}}^{2} \leq C\left(\int_{0}^{t} \sup _{x}|\bar{\rho}(s, x)|^{2} d s+\|\bar{u}\|_{H^{\frac{11}{4}, \frac{11}{8}\left(P_{t}\right)}}^{2}+\|\bar{v}\|_{H^{\frac{11}{4}, \frac{11}{8}\left(P_{t}\right)}}^{2}\right),
\end{aligned}
$$

and

$$
\left\|\bar{g}_{e}\right\|_{H^{\frac{9}{8}\left(P_{t}\right)}}^{2} \leq C\left(\|\bar{u}\|_{H^{\frac{11}{4}, \frac{11}{8}}\left(P_{t}\right)}^{2}+\|\bar{v}\|_{H^{\frac{11}{4}, \frac{11}{8}\left(P_{t}\right)}}^{2}\right) .
$$

(iv) Again,

$$
\begin{aligned}
\|\bar{u}\|_{H^{\frac{11}{4}, \frac{11}{8}\left(P_{t}\right)}} \leq C\left(\left\|\bar{f}_{e}\right\|_{H^{\frac{3}{4}, \frac{3}{8}\left(P_{t}\right)}}+\left\|\bar{g}_{e}\right\|_{H^{\frac{5}{8}\left(\Sigma_{t}\right)}}\right), \\
\|\bar{v}\|_{H^{\frac{11}{4}, \frac{11}{8}\left(P_{t}\right)}} \leq C\left\|\bar{f}_{i}\right\|_{H^{\frac{3}{4}, \frac{3}{8}\left(P_{t}\right)}} .
\end{aligned}
$$

(v) Then it is shown that

$$
\begin{aligned}
& \left\|\bar{f}_{e}\right\|_{H^{\frac{3}{4}, \frac{3}{8}\left(P_{t}\right)}}^{2} \leq C\left(\int_{0}^{t} \sup _{x}|\bar{\rho}(s, x)|^{2} d s+\|\bar{u}\|_{H^{\frac{7}{4}, \frac{7}{8}\left(P_{t}\right)}}^{2}+\|\bar{v}\|_{H^{\frac{7}{4}, \frac{7}{8}\left(P_{t}\right)}}^{2}\right), \\
& \left\|\bar{f}_{i}\right\|_{H^{\frac{3}{4}, \frac{3}{8}\left(P_{t}\right)}}^{2} \leq C\left(\int_{0}^{t} \sup _{x}|\bar{\rho}(s, x)|^{2} d s+\|\bar{u}\|_{H^{\frac{7}{4}, \frac{7}{8}\left(P_{t}\right)}}^{2}+\|\bar{v}\|_{H^{\frac{7}{4}, \frac{7}{8}\left(P_{t}\right)}}^{2}\right),
\end{aligned}
$$

and

$$
\left\|\bar{g}_{e}\right\|_{H^{\frac{5}{8}\left(P_{t}\right)}}^{2} \leq C\left(\|\bar{u}\|_{H^{\frac{7}{4}, \frac{7}{8}\left(P_{t}\right)}}^{2}+\|\bar{v}\|_{H^{\frac{7}{4}, \frac{7}{8}\left(P_{t}\right)}}^{2}\right) .
$$

(vi) Again,

$$
\begin{aligned}
& \|\bar{u}\|_{H^{\frac{7}{4}, \frac{7}{8}\left(P_{t}\right)}} \leq C\left(\left\|\bar{f}_{e}\right\|_{L^{2}\left(P_{t}\right)}+\left\|\bar{g}_{e}\right\|_{H^{\frac{1}{4}\left(\Sigma_{t}\right)}}\right), \\
& \|\bar{v}\|_{H^{\frac{7}{4}, \frac{7}{8}\left(P_{t}\right)}} \leq C\left\|\bar{f}_{i}\right\|_{L^{2}\left(P_{t}\right)} \text {. }
\end{aligned}
$$


(vii) It is then shown that

$$
\begin{aligned}
& \left\|\bar{f}_{e}\right\|_{L^{2}\left(P_{t}\right)}^{2} \leq C\left(\int_{0}^{t} \sup _{x}|\bar{\rho}(s, x)|^{2} d s+\|\bar{u}\|_{L^{2}\left(0, t ; H^{1}(\Omega)\right)}^{2}+\|\bar{v}\|_{L^{2}\left(0, t ; H^{1}(\Omega)\right)}^{2}\right), \\
& \left\|\bar{f}_{i}\right\|_{L^{2}\left(P_{t}\right)}^{2} \leq C\left(\int_{0}^{t} \sup _{x}|\bar{\rho}(s, x)|^{2} d s+\|\bar{u}\|_{L^{2}\left(0, t ; H^{1}(\Omega)\right)}^{2}+\|\bar{v}\|_{L^{2}\left(0, t ; H^{1}(\Omega)\right)}^{2}\right),
\end{aligned}
$$

and

$$
\left\|\bar{g}_{e}\right\|_{H^{\frac{1}{4}\left(P_{t}\right)}}^{2} \leq C\left(\|\bar{u}\|_{H^{1, \frac{1}{2}\left(P_{t}\right)}}^{2}+\|\bar{v}\|_{H^{1, \frac{1}{2}\left(P_{t}\right)}}^{2}\right) .
$$

(viii) Finally, the estimation $\|\bar{u}\|_{H^{1, \frac{1}{2}}} \leq C\left(\int_{0}^{t} \sup _{x}|\bar{\rho}|^{2}\right)^{\frac{1}{2}}$ is obtained.

Since $\rho$ is the first component of the solution of the Euler system, it follows from 4.34 and 4.35 that

$$
\mathcal{U}(t)^{2} \leq c \int_{0}^{t} \sup _{x} \bar{\rho}^{2}(s, \cdot)^{2} d s,
$$

and so, $\mathcal{U}(t)^{2} \leq c \int_{0}^{t} \mathcal{U}(s)^{2} d s$. Gronwall's lemma implies that $\mathcal{U}=0$ so that $\bar{u}=\bar{v}=0$. Hence the uniqueness of the solution is proved. This ends the proof of Theorem 5 .

\section{REFERENCES}

[1] J.-P. Boeuf and A. Merad, Fluid and Hybrid Models of Non-Equilibrium Discharges, Plasma Processing of Semiconductors, NATO ASI Series E: Applied Sciences, Vol. 336, Ed. P. F. Williams, Kluwer Academic Publisher, 1997, pp. 291-320

[2] W. S. Boyle, P. Kisliuk, and L. H. Germer, Electrical breakdown in high vacuum, J. Appl. Phys. 26, 720-725 (1955)

[3] H. Brezis, Analyse Fonctionnelle, Masson, 1987

[4] C. Cercignani, The Boltzmann Equation and its Applications, Springer-Verlag, New York, 1988

[5] F. Chen, Plasma Physics and Controlled Fusion, Plenum Publishing Corporation, Second Edition, 1984

[6] M. Cho and D. E. Hastings, Dielectric charging process and arcing rate of high voltage solar arrays, J. Spacecraft and Rockets 28, 698-706 (1990)

[7] P. Degond and B. Lucquin, Transport coefficient of plasmas and disparate mass binary gases, Math. Models Methods Appl. Sci. 6, 405-436 (1996)

[8] D. Gilbarg and N. S. Trudinger, Elliptic Partial Differential Equations of Second Order, SpringerVerlag, New York, 1983

[9] P. Grisvard, Caracterisation de quelques espaces d'interpolation, Arch. Rat. Mech. Anal. 25, 40-63 (1967)

[10] D. E. Hastings, M. Cho, and H. Kuninaka, Arcing rates for high voltage solar arrays: Theory, experiment and prediction, J. Spacecraft and Rockets, 538-554 (1992)

[11] I. Katz and D. B. Snyder, Mechanism for spacecraft charging, initiated destruction of solar arrays in GEO, AIAA, 98-1002, 36th Aerospace Sciences Meeting and Exhibit, Reno, NV, 1998

[12] J. L. Lions and E. Magenes, Problèmes aux Limites non Homogenes, Volumes 1 and 2, Dunod, 1968

[13] Li Ta-Tsien and Yu Wen-ci, Boundary Value Problems for Quasilinear Hyperbolic Systems, Math. Series V, Duke Univ., Durham, NC, 1985

[14] J. P. Marque, Phenomenology of e-irradiated polymer breakdown, Vacuum, 39, no. 5, 443-452 (1989)

[15] D. Payan, Modèlisation des fils flottants; caractérisation des décharges électrostatiques de l'âme d'un fil, Internal note, CNES NT-I-93-11079/ACE, 1993

[16] Y. Raizer, Gas Discharge Physics, Springer-Verlag, New York, 1991

[17] T. Seidman and G. Troianiello, Time-dependent solutions of a nonlinear system arising in semiconductor theory, in Nonlinear Analysis, Theory, Methods and Applications, Vol. 9, No. 11, 1985, pp. 1137-1157 
[18] T. Seidman and G. Troianiello, Time-dependent solutions of a nonlinear system arising in semiconductor theory-II. Boundedness and periodicity, Nonlinear Analysis, Theory, Methods and Applications 10, 491-502 (1986)

[19] F. Severin, Etude théorique et simulation numérique de décharge électrique dans le vide, Ph.D., ENPC, France, 1998

[20] F. Severin and J. P. Marque, Numerical simulation of an electrical discharge in vacuum, preprint, 1999 